\title{
First Order Phase Transitions in Lattice and Continuous Systems: Extension of Pirogov-Sinai Theory *
}

\author{
J. Bricmont ${ }^{1}$, K. Kuroda ${ }^{2}$, and J. L. Lebowitz ${ }^{3}$ \\ 1 Institut de Physique Théorique, chemin du Cyclotron, 2, B-1348 Louvain-la-Neuve, Belgium \\ 2 Department of Mathematics, Keio University, Hiyoshi 3-14-1, Kohoku-ku, Yokohama 223, \\ Japan \\ 3 Department of Mathematics and Physics, Rutgers University, New Brunswick, NJ 08903, USA
}

\begin{abstract}
We generalize the notion of "ground states" in the Pirogov-Sinai theory of first order phase transitions at low temperatures, applicable to lattice systems with a finite number of periodic ground states to that of "restricted ensembles" with equal free energies. A restricted ensemble is a Gibbs ensemble, i.e. equilibrium probability measure, on a restricted set of configurations in the phase space of the system. When a restricted ensemble contains only one configuration it coincides with a ground state. In the more general case the entropy is also important.

An example of a system we can treat by our methods is the $q$-state Potts model where we prove that for $q$ sufficiently large there exists a temperature at which the system coexists in $q+1$ phases; $q$-ordered phases are small modifications of the $q$ perfectly ordered ground states and one disordered phase which is a modification of the restricted ensemble consisting of all "perfectly disordered" (neighboring sites must have different spins) configurations. The free energy thus consists entirely of energy in the first $q$-restricted ensembles and of entropy in the last one.

Our main motivation for this work is to develop a rigorous theory for phase transitions in continuum fluids in which there is no symmetry between the phases, e.g. the liquid-vapour phase transition. The present work goes a certain way in that direction.
\end{abstract}

\section{Introduction}

In 1936, Peierls [1] invented an argument to show that the Ising model on a $d$-dimensional lattice, $d \geqq 2$, with nearest neighbor ferromagnetic interactions has spontaneous magnetization at low enough temperatures. The system can exist in either $\mathrm{a}+$ or a - phase: the signature of a first order phase transition. Dobrushin [2] and Griffiths [3] later made the argument mathematically precise. This

* Supported in part by NSF Grant N DMR81-14726-02 
argument has been extended and new methods have been invented, to prove phase coexistence, i.e. first order phase transitions, in a great variety of systems.

These methods fall into various categories. Most require however that, like in the original Ising model, the different phases be related by a symmetry of the Hamiltonian. This includes Ruelle's [4] extension of the Peierls' argument to the symmetric continuum Widom-Rowlinson model. An outstanding exception is the Pirogov-Sinai theory [5] and its extensions [6-15]. This theory applies to general lattice models of the following type: The system is described by occupation (or spin) variables which can take on a finite number of values at each site of a $d$-dimensional regular lattice, $d \geqq 2$. The particles interact with arbitrary finite range periodic potentials, e.g. an Ising system with one, two, and three spin interactions. The Hamiltonian of the system $H_{0}$ has $n$ periodic ground states, $n$ finite, and there is a non-zero minimum energy per unit interface, or "contour," separating two ground states: the Peierls' condition.

Pirogov and Sinai study the structure of the phase diagram of the Hamiltonian $H_{\underline{\mu}}$,

$$
H_{\underline{\mu}}=H_{0}+\sum_{i=1}^{n-1} \mu_{i} H_{i}
$$

in the $n-1$-dimensional parameter space of "chemical potentials" $\mu_{1}, \ldots, \mu_{n-1}$. The $H_{i}$ are perturbations which lift the degeneracy of $H_{0}$ and produce, in $\mu$-space, the following topological structure of the ground states of $H_{\mu}$ : There are $n$-lines emanating from the origin on which $H_{\mu}$ has $n-1$ periodic ground states, two dimensional surfaces bounded by pairs of these lines on which there are $n-2$ ground states, etc.

The theorem then states that, at sufficiently low temperatures, the phase diagram perfectly mimics the above structure. There is a point $\mu^{(0)}$ at which there are $n$ periodic phases etc.

This paper as well as our previous work [12] is an extension of Pirogov-Sinai theory having as its primary goal the understanding of phase transitions in continuum fluids in which there is no symmetry between the phases, e.g. the liquidvapour phase transition. While we have not yet succeeded in doing this we believe that the present work goes a certain way in that direction; see discussion at end. Other generalizations, some of them closely related to our work, have been carried out recently by various authors [11,13-15]. In particular Imbrie has extended the work of Glimm, Jaffe, and Spencer on phase transitions in quantum field theories by making a powerful generalization of Pirogov-Sinai theory to such systems [7].

Example. A simple example illustrates the kind of extensions of the Pirogov-Sinai theory we make here. Consider a lattice (or continuum) gas with 3 species of particles: $A, B$, and $C$. The interaction consists simply of a hard-core of radius $R$ between an $A$ particle and a $B$ or $C$ particle. There are no other interactions between the particles. There is thus a trivial $B-C$ symmetry. The species have an activity $z_{A}, z_{B}$, and $z_{C}$. From the point of view of the Pirogov-Sinai theory the limit $z_{A}=z_{B}=z_{C} \rightarrow \infty$ leads to an infinity of ground states given by either the configuration $\omega_{x}=A \forall x$, or by the set of configurations where $\omega_{x} \in\{B, C\} \forall x$.

Since there is an infinite number of "ground states" the standard theory does not apply. It is intuitively clear however that for large activities, there will be a 
separation between an $A$-dominated phase and a $B-C$ dominated phase but no separation inside the $B-C$ mixture, since there is no interaction to induce it. This suggests that it may be possible to group an infinity of degenerate ground states into a finite set of classes with each class giving rise to one equilibrium state (phase) at low temperatures.

In fact our extension of the Pirogov-Sinai theory consists in the replacement of the notion of ground states by measures over suitable subsets of phase space called "restricted ensembles": ${ }^{1}$ In the above example these are the single configuration $\omega_{x}=A \forall x \in \mathbb{Z}^{d}$ (which is a ground state in the usual sense) and the Gibbs measure (with no interactions) over the set of configurations

$$
\left\{\omega \mid \omega_{x} \in\{B, C\} \forall x \in \mathbb{Z}^{d}\right\} .
$$

For large $z_{B}, z_{C}$ and suitable $z_{A}$ we shall have two phases whose typical configurations will belong to one of these two subsets with small islands of the opposite set (and of empty regions).

Moreover, the value of $z_{A}$ where this occurs is asymptotically $\left(z_{B}, z_{C} \rightarrow \infty\right)$ close to the values where the free energies of the restricted ensembles are equal: $z_{A} \cong z_{B}+z_{C}$. Thus we have the same picture as in the usual Pirogov-Sinai theory: restricted ensemble $\rightarrow$ pure phases. The correspondence is as follows: in the Pirogov-Sinai theory all ground states have the same energy per unit volume; here the requirement is that the different restricted ensembles have the same free energy per unit volume. Then, for nearby values of the parameters specifying the Hamiltonian $H_{\underline{\mu}}$ we find a number of phases corresponding to the number of restricted ensembles. One then also obtains a complete phase diagram in the vicinity of this point.

General Framework. Let us now describe the general framework in which this example fits. First of all, it is not necessary that all configurations of $B-C$ mixtures have the same energy. There can be interactions between the $B-C$ particles themselves. What we require about their restricted ensembles is a diluteness property. By this we mean that if we look at the system restricted to such an ensemble it has to be in a single phase and satisfy (very) strong clustering properties. Such properties usually follow from existence of convergent expansions in a small parameter characterizing the "dilute" concentration of defects; hence the name.

In the case of a restricted ensemble being a single configuration (i.e. a ground state) these properties are trivial, so our restricted ensembles properly generalize the notion of ground state. The $B-C$ mixture in the example above is also dilute, since the $B$ and $C$ particles are independent, but there could be some weak interactions between them without destroying the diluteness property. We prove this for our example as well as for other models that we treat by means of some convergent expansion (Mayer, high-temperature, etc.). However, one can formulate the general theory by assuming clustering properties in the restricted ensembles that, although they are hard to check, should hold beyond the radius of

1 A similar idea was introduced in Field Theory [7] when a contour expansion was combined with an expansion around each minimum of the potential. The set of field configurations close to a minimum corresponds to our notion of "restricted ensemble" 
convergence of these expansions. They are probably true generally for systems in the one-phase region away from critical points.

In addition to the diluteness hypothesis, we have to assume a suitable Peierls' condition: consider a set of configurations such that in one region $\Lambda_{1}$ the system is in one restricted ensemble and in a different region $\Lambda_{2}$ is in a different restricted ensemble. Then we assume that $\Lambda_{1}$ and $\Lambda_{2}$ have to be separated by a region, called contour, where the system is in none of the restricted ensembles. Moreover, the free energy of the system, given this specification, is higher than the one of the system which is in the same restricted ensemble everywhere by an amount equal to the size of the contour times a large constant.

Finally, we assume that one can find a point in parameter space where the free energies per unit volume of the different restricted ensembles coincide. Then we also want a set of perturbations that completely "lifts the degeneracy of the restricted ensembles." Free energy degeneracy of restricted ensemble is thus similar to ground state degeneracy in the Pirogov-Sinai theory.

An interesting example that we can analyze, where the entropy is essential, is the $q$-state Potts model (on $\mathbb{Z}^{d}, d \geqq 2$ ) studied by Kotecky and Shlosman [16] using reflection positivity methods. Here for $q$ large we have $q+1$ restricted ensembles; $q$ of them are "ordered," each corresponding to a single configuration with all spins equal and the $(q+1)^{\text {th }}$ ("disordered") consists of all configurations where all pairs of adjacent spins are unequal. All these restricted ensembles have the same free energy for $\beta$ approximately equal to $\frac{1}{d} \log q$. For the ordered ensemble this free energy is just their energy (one configuration $=0$ entropy) while for the disordered one it is a pure entropy factor. Moreover, for $q$ large, all these ensembles are dilute and Peierls' Condition holds: that is, all intermediate situations between total order and total disorder have a higher free energy.

Technically the main idea of the Pirogov-Sinai theory is to realize the distribution of "outer contours" with the help of a suitable "contour model." In the original theory the contours in this model are characterized by one body potentials and interact only by hard core exclusion, i.e. they cannot overlap. In our case however we have to consider contour models with interactions and we use the diluteness hypothesis to prove that these models enjoy all the usual properties of contour models.

Outline. In Sect. II we state the theorems that we can prove. We consider first particular models (Widom-Rowlinson models, Potts model) and then the general framework outlined above. Next, we prove our results, starting with the general formulation and then showing that the models are special cases of it. In the last section we discuss an alternative approach to our general results and indicate some extensions.

\section{Models and Main Results}

We start by listing several models for which we shall prove the coexistence of phases. These include both continuum and lattice models. We then develop a general framework which extends the Pirogov-Sinai theory and in which all previous models fit. We always assume that the space dimension $d \geqq 2$. 


\section{A. Models}

1) $A-B-C$ Model on a Lattice. As described in the Introduction, we associate to each site $x \in \mathbb{Z}^{d}$ a "spin" variable $\omega_{x}$ taking four values, $A, B, C$, and 0 , where $\omega_{x}=0$ means there is no particle at site $x \in \mathbb{Z}^{d}$. The set of configurations is denoted by $\Omega$ and the restriction of $\omega \in \Omega$ to $X \subset \mathbb{Z}^{d}$ by $\omega_{X}$.

The interaction consists of two parts: 1) A hard-core interaction with radius $R$ between an $A$-particle and a $B$ or $C$ particle.

2) A finite range interaction between $B$ and $C$ particles given by

$$
H_{\Lambda}(\omega)=\sum_{X \subset A} J_{X}\left(\omega_{X}\right), \quad|X| \geqq 2,
$$

where $J=\left\{J_{X}(\cdot)\right\}_{X \subset \mathbb{Z}^{d}}$, is an interaction which satisfies,

i) $J$ is periodic,

ii) $J$ is finite range, i.e. $J_{X} \equiv 0$ if $\operatorname{diam}(X) \geqq \bar{R}$,

iii) $J_{X}\left(\omega_{X}\right)=0$ unless $\omega_{x} \in\{B, C\}$ for all $x \in X$.

We denote the fugacity of $A$-particles by $z_{A}$ and the fugacity of $B$ and $C$ particles, taken equal for simplicity, by $z_{0}$. We prove the phase coexistence of an $A$-phase and a $B-C$ mixture phase when $\beta$ is sufficiently small and $z_{A}, z_{0}$ are large. The "restricted partition functions" $Z^{A}(\Lambda), Z^{B, C}(\Lambda)$, corresponding to Gibbs states in the restricted ensembles, are given by

$$
\begin{gathered}
Z_{R}^{A}(\Lambda)=z_{A}^{|\Lambda|}, \\
Z_{R}^{B, C}(\Lambda)=z_{0}^{|\Lambda|} \cdot \sum^{B, C} \exp \{-\beta H \Lambda(\omega)\},
\end{gathered}
$$

where, in $\sum^{B, C}$, we sum over all $\omega_{\Lambda} \in \Omega_{\Lambda}$ such that $\omega_{x} \in\{B, C\}$ for all $x \in \Lambda$.

The restricted free energies $f_{A}$ and $f_{B, C}$ are given by

$$
f_{A}=\ln z_{A}, \quad f_{B, C}=\lim _{A \rightarrow \mathbb{Z}^{d}} \frac{1}{|\Lambda|} \ln Z_{R}^{B, C}(\Lambda) .
$$

We now note that, by employing the high temperature expansion (see Appendix 1), we have, for sufficiently small $\beta$,

$$
\ln Z_{R}^{B, C}(\Lambda)=|\Lambda|\left(\ln z_{0}+h(\beta)\right)+\Delta(\beta, \Lambda),
$$

with $|\Delta(\beta, \Lambda)| \leqq g(\beta)|\partial \Lambda|, g(\beta) \rightarrow 0$ as $\beta \rightarrow 0$, and $h(\beta)=\ln 2+O(\beta)$.

Hence $f_{A}=f_{B, C}$ if $z_{A}=z_{0} \exp (h(\beta))$. Our results about phase coexistence can now be stated as follows:

Theorem 1. There exists $\bar{\beta}$ and $\bar{z}_{0}$ such that, for each $\beta, z_{0}, \beta<\bar{\beta}, z_{0}>\bar{z}_{0}$, and for some $z_{A}\left(\beta, z_{0}\right)$, close to $z_{0} \exp (h(\beta))$, at least two different extremal Gibbs states $p^{A}(\cdot)$ and $p^{B, C}(\cdot)$ coexist. The typical configurations of $p^{A}$ (respectively $p^{B, C}$ ) consists of a sea of $A$ particles (respectively $B$ or $C$ particles) with small islands of $B$ or $C$ particles (respectively $A$ particles).

2) $A-B-C$ Model in the Continuum.

$1^{\circ}$ ) In this version of the $A-B-C$ model, there are $A, B$, and $C$ particles with fugacities $z_{A}, z_{B}$, and $z_{C}$ in the continuum. There is a hard core interaction with radius $R_{0}$ between an $A$ and a $B$ or a $C$ particle. In addition we have four types of 
pair interactions $\phi_{A, A}, \phi_{B, B}, \phi_{C, C}$, and $\phi_{B, C}$, all of which satisfy the following condition:

Condition A. $\phi_{\alpha \beta}(r)$ has finite range $\bar{R}$ and either $\phi_{\alpha \beta}(r)=\infty$ for $r \leqq r_{\alpha \beta}$ and is bounded for $r>r_{\alpha \beta}$ or $\phi_{\alpha \beta}(r)=0$.

Notice that in both cases $\phi_{\alpha \beta}(r)$ satisfies the following strong stability condition: there exists $0<B<\infty$ such that for any admissible configuration $\left\{x_{j}\right\}_{j=1}^{n}$, i.e. compatible with the hard core condition, and any $i \in\{1,2, \ldots, n\}$, we have

$$
\left|\sum_{j=1, j \neq i}^{n} \phi_{\alpha \beta}\left(x_{i}-x_{j}\right)\right| \leqq B<\infty .
$$

Theorem 2. Assume that the $\phi$ 's satisfy condition $A$. Then there exists $c<\infty$ such that for each $z_{A} \geqq c$ there exists $\beta_{0}$ and $r_{0}$ with the property that, if $0 \leqq \beta \leqq \beta_{0}$ and $r_{\alpha \beta} \leqq r_{0}$, we can find values $z_{B}(\beta)$ and $z_{C}(\beta)$ for which at least two different extremal Gibbs states $p^{A}(\cdot)$ and $p^{B, C}(\cdot)$ coexist. The typical configurations of $p^{A}, p^{B, C}$ are as in Theorem 1.

Remark. Contrary to the situation in Theorem $1, \beta_{0}$ and $r_{0}$ here depend on $z_{A}$. The larger $z_{A}$ the smaller $\beta_{0}, r_{0}$. This is necessary in order to satisfy our diluteness hypothesis in the restricted ensemble.

$2^{\circ}$ ) We may consider another version of this model, in which there is coexistence between an $A$-rich phase and a $B$-rich phase each of which contains a small number of $C$-particles. There is a hard-core interaction between $A$ and $B$ particles and pair interactions between all other pairs of particles $\phi_{A, A}, \phi_{B, B}, \phi_{C, C}$, $\phi_{A, C}$, and $\phi_{B, C}$ all of which satisfy condition $A$.

Under the above assumptions on interactions we have the following result.

Theorem 3. There exists $c<\infty$ such that, for $z_{A} \geqq c$, there exist $\beta_{0}, r_{0}$, and $z_{0}$ with the property that, if $0 \leqq \beta \leqq \beta_{0}, 0 \leqq z_{C} \leqq z_{0}$ and $r_{\alpha \beta} \leqq r_{0}$, we can find a value $z_{B}\left(\beta, z_{A}, z_{C}\right)$ for which at least two different extremal Gibbs measures $p^{A}(\cdot)$ and $p^{B}(\cdot)$ coexist.

3) r-State Continuum Widom-Rowlinson Model with Interactions. Here we consider the same framework as in [12] but now allow some interactions among particles of the same type. There are $r$ species of particles with fugacities $z_{\alpha}, \alpha \in S$ $=\{0,1, \ldots, r-1\}$. The interaction between $\alpha$ and $\beta$ particles is via a hard-core

$$
\phi_{\alpha, \beta}(r)= \begin{cases}\infty & \text { if }|r| \leqq R_{\alpha, \beta} \\ 0 & \text { otherwise } .\end{cases}
$$

The pair interaction $\phi_{\alpha, \alpha}$ among particles of type $\alpha$ satisfies condition $A$ for each $\alpha \in S$.

Let us fix the fugacity $z_{0}$ sufficiently large, put

$$
\hat{z}=\left(z_{1}, \ldots, z_{r-1}\right) \in \mathbb{R}^{r-1}
$$

and consider the parameter space:

$$
U\left(z_{0}, \varepsilon\right)=\left\{\hat{z} \in \mathbb{R}^{r-1} ;|\hat{z}-\hat{z}(O)| \leqq \varepsilon z_{0}\right\},
$$

where $\hat{z}(O)=\left(z_{0}, \ldots, z_{0}\right)$ and $|\hat{z}|=\operatorname{Max}_{1 \leqq i \leqq r-1}\left|z_{i}\right|$. 
The following result is the extension of the main theorem of [12].

Theorem 4. Under the above hypotheses, there exists an $\varepsilon>0$ and a $c<\infty$ such that, for $z_{0} \geqq c$, there exist $\beta_{0}, r_{0}$ with the property that, if $0 \leqq \beta \leqq \beta_{0}$, and $r_{\alpha \alpha} \leqq r_{0}$ for $\alpha=0, \ldots, r-1$, the phase diagram in $U\left(z_{0}, \varepsilon\right)$ is as follows:

1) There exists a point $\hat{z}_{0} \in U\left(\varepsilon, z_{0}\right)$ for which at least $r$ different extremal Gibbs states coexist.

2) There exist $r$ lines $\gamma_{\alpha}, \alpha=0, \ldots, r-1$, starting from $\hat{z}_{0}$, for which at least $r-1$ extremal Gibbs states $\left\{p_{i}\right\}, i \in S \backslash\{\alpha\}$, coexist.

3) In general, there exist $k$-dimensional open surfaces $\gamma_{A} \subset U\left(\varepsilon, z_{0}\right), A \subset S$, $\# A=k$, the boundary of $\gamma_{A}$ consists of $\bar{\gamma}_{A \backslash\{\alpha\}}, \alpha \in A$, and at least $r-k$ extremal Gibbs states $\left\{p_{i}\right\}, i \in S \backslash A$ coexist on $\gamma_{A}$.

4) q-State Potts Model. This model is defined as follows: at each site $x \in \mathbb{Z}^{d}, d \geqq 2$, there is a variable $S_{x} \in\{1,2, \ldots, q\}$, and the Hamiltonian is given by

$$
H_{\Lambda}(S)=-\sum_{\langle x, y\rangle c \Lambda} \delta_{S_{x}, S_{y}},
$$

where $\langle x, y\rangle$ is a nearest neighbor pair of sites in $\mathbb{Z}^{d}$.

By using reflection positivity Kotecky and Shlosman [16] proved the phase coexistence between "ordered phases" and the "disordered phase." Here ordered phases correspond to the configurations $\omega^{i}, i=1, \ldots, q$, given by

$$
\omega^{i}(t)=i \text { for all } t \in \mathbb{Z}^{d},
$$

and the disordered phase corresponds to the subset $\Omega^{D}$ of $\Omega$ given by

$$
\Omega^{D}=\{\omega: \omega(t) \neq \omega(s) \text { for all nearest neighbor sites }(t, s)\} .
$$

Let us note again that the energy plays the central role in the ordered phase while the entropy is all important in the disordered phase.

For this system we shall first prove the properties of contour correlation functions by employing the Pirogov-Sinai method and then prove coexistence of the corresponding phases. The advantage of our method is that it doesn't require reflection positivity. We can obtain information about contour correlation functions and asymptotic estimates on the transition temperature. ${ }^{2}$ One could also consider more general interactions and more general phase diagrams, including several perturbations; see also $[14,15]$ for alternative proofs of Theorem 5.

Theorem 5. There exists a $q_{0}$ such that for any $q>q_{0}$ we can find a value $\beta(q)$ with $\left|\beta(q)-\frac{1}{d} \ln q\right|<O\left(q^{-(1-1 / d)}\right)$ at which at least $q+1$ different extremal translation invariant Gibbs states $p^{1}(\cdot), \ldots, p^{q}(\cdot)$ and $p^{D}(\cdot)$ coexist. $p^{i}(\cdot), i \in S$, and $p^{D}(\cdot)$ satisfy the estimates:

$$
\begin{gathered}
p^{i}\left(\omega\left(t_{0}\right)=i\right)>\frac{1}{2}, \\
p^{D}\left(\omega\left(t_{0}\right)=j\right)=q^{-1}, \quad \text { all } j, \text { and } p^{D}\left(\omega\left(t_{0}\right) \neq \omega\left(t_{1}\right)\right)>\frac{1}{2},
\end{gathered}
$$

where $\left(t_{0}, t_{1}\right)$ is any pair of nearest neighbor sites.

2 Related estimates have been obtained by R. Israël (private communication) 


\section{B. General Framework}

(1) Description of the System. We consider a system consisting of $s$ species of particles. We discuss it in the continuum but a simple modification of this scheme allows us to cover the lattice case, too. We have pair interactions $\phi_{\alpha, \beta}$ satisfying condition $A$. More general many-body interactions, for which (2.6) below is finite, can be included as well.

The grand canonical partition function in a volume $\Lambda \subset \mathbb{R}^{d}$ is given by

$$
Z(\Lambda)=\sum_{0}^{\infty} \frac{z_{1}^{n_{1}} \ldots z_{s}^{n_{s}}}{n_{1} ! \ldots n_{s} !} \cdot \int_{\Lambda} \exp \left[-\beta \hat{H}\left(\underline{x}_{n_{1}}^{1}, \ldots, \underline{x}_{n_{s}}^{s}\right)\right] I\left(\underline{x}_{n_{1}}^{1}, \ldots, \underline{x}_{n_{s}}^{s}\right) d \underline{x}_{n_{1}}^{1} \ldots d \underline{x}_{n_{s}}^{s},
$$

where $\underline{x}_{n_{i}}^{i}=\left(x_{1}^{i}, \ldots, x_{n_{i}}^{i}\right)$ is the configuration of the $i^{\text {th }}$ type of particles, and

$$
\hat{H}\left(\underline{x}_{n_{1}}^{1}, \ldots, \underline{x}_{n_{s}}^{s}\right)=\sum_{\alpha, \beta=1}^{s} \sum_{i, j} \phi_{\alpha, \beta}\left(x_{i}^{\alpha}-x_{j}^{\beta}\right)+\ldots
$$

The ... stands for the other many-body interactions and $I\left(\underline{x}_{n_{1}}^{1}, \ldots, \underline{x}_{n_{s}}^{s}\right)$ is the restriction coming from the hard-core interactions:

$$
I\left(\underline{x}_{n_{1}}^{1}, \ldots, \underline{x}_{n_{s}}^{s}\right)= \begin{cases}0 & \text { if there exist } x_{k}^{i} \text { and } x_{l}^{j} \text { with }\left|x_{k}^{i}-x_{l}^{j}\right| \leqq r_{i j}, \\ 1 & \text { otherwise }\end{cases}
$$

We abbreviate $\xi=\left(\underline{x}_{n_{1}}^{1}, \ldots, \underline{x}_{n_{s}}^{s}\right)$ and define,

$$
H(\xi)=\beta \hat{H}(\xi)+\sum_{\alpha=1}^{s} h_{\alpha} n_{\alpha}
$$

$h_{\alpha}=\log z_{\alpha}$, and we write

$$
d v_{\Lambda}(\xi)=I(\xi) d \tilde{v}_{\Lambda}(\xi)
$$

where $\tilde{v}_{\Lambda}(\xi)$ is the natural measure on $\bigcup_{\left\{n_{\alpha}\right\}} \prod_{\alpha=1}^{s} \Lambda^{n_{\alpha}}$ given by

$$
\sum_{\left\{n_{\alpha}\right\}} \prod_{\alpha=1}^{s}\left(n_{\alpha} !\right)^{-1} d \underline{x}_{n_{\alpha}}^{\alpha} .
$$

The partition function can now be written as

$$
Z(\Lambda)=\int d v_{\Lambda}(\xi) \exp (-H(\xi))
$$

Let the set of locally finite configurations compatible with the hard-core conditions be denoted by $\Omega$. We write $\omega_{\Lambda}$ to denote the restriction of $\omega \in \Omega$ to $\Lambda$ and $\Omega_{\Lambda}=\left\{\omega_{\Lambda} \mid \omega \in \Omega\right\}$ for the configuration space in $\Lambda$.

We can introduce boundary conditions by fixing $\omega \in \Omega_{\bar{\Lambda}}, \bar{\Lambda}=\mathbb{R}^{d} \backslash \Lambda$. This defines $H(\xi \mid \omega), I(\xi \mid \omega)$, and $\nu_{\Lambda}(\xi \mid \omega)$.

We introduce a norm on $H$ :

$$
\|H\|=\sup \frac{|H(\xi)|}{n(\xi)}
$$

where $n(\xi)=\sum_{\alpha=1}^{s} n_{\alpha}(\xi)$ is the total number of particles in $\xi$. The sup is taken over all configurations with a finite number of particles that are compatible with the hardcore conditions. 
In the sequel we shall assume that $\|H\|$ is finite.

(2) Restricted Ensembles. We cover $\mathbb{R}^{d}$ with a grid $\mathbb{Z}^{d}$ of lattice spacing 1 (i.e. we choose this spacing as a unit of length) and we denote by $C_{i}, i \in \mathbb{Z}^{d}$ the unit cell of the dual lattice centered at $i: C_{i}=\left\{x \in \mathbb{R}^{d}\left|\max _{\alpha}\right| x_{\alpha}-i_{\alpha} \mid \leqq \frac{1}{2}\right\}$. These cells will be called "elementary cubes." We assume that $\Omega_{C_{0}}$ can be partitioned as follows:

$$
\Omega_{C_{0}}=\bigcup_{q=1}^{r} \Omega_{0}^{q} \cup \bar{\Omega}_{0},
$$

and we define $\Omega_{i}^{q}, \bar{\Omega}_{i}$ as the set of configurations obtained by translating $\Omega_{0}^{q}, \bar{\Omega}_{0}$. The configurations in $\Omega_{i}^{q}, q=1, \ldots, r$ will be our generalized ground states, while the configurations in $\bar{\Omega}_{i}$ will enter implicitly into the definition of contours.

We define the sets of configurations in our restricted ensembles

$$
\Omega^{q}=\left\{\omega \in \Omega \mid \omega_{C_{\imath}} \in \Omega_{i}^{q} \forall i \in \mathbb{Z}^{d}\right\}, \text { and } \Omega_{\Lambda}^{q}=\left\{\omega_{\Lambda} \mid \omega \in \Omega^{q}\right\} .
$$

By an abuse of language we often refer to $\Omega^{q}$ as the $q^{\text {th }}$ restricted ensemble.

Now we illustrate this notion with a few examples:

$\left.1^{\circ}\right)$ Ising Model. Let $C_{0}$ consist of one site in $\mathbb{Z}^{d}$. Now we have two restricted ensembles $\Omega^{1}$ and $\Omega^{-1}$ given by $\Omega^{1}=\left\{\omega^{+}\right\}$and $\Omega^{-1}=\left\{\omega^{-}\right\}$, where $\omega^{ \pm}(t)= \pm 1$ for all $t \in \mathbb{Z}^{d} . \bar{\Omega}_{i}$ is empty.

$\left.2^{\circ}\right) A-B-C$ Model. For the lattice model, let $C_{0}$ be one lattice site. We have now restricted ensembles $\Omega_{A}=\left\{\omega_{A}\right\}, \omega_{A}(t) \equiv A, \forall t \in \mathbb{Z}^{d}$, and $\Omega_{B, C}=\{\omega \in \Omega ; \omega(t)=B$ or $C$ for all $\left.t \in \mathbb{Z}^{d}\right\}$, and $\bar{\Omega}_{i}$ is the configuration with no particle at site $i$.

For the continuum model one chooses a box $C_{0}$ such that if there is one $A$-particle in $C_{0}$ we cannot put a $B$ or $C$-particle in any box $C_{i}$ with $|i|<2$. However, an $A$ particle is allowed in each $C_{i}$ and if there is a $B$ or $C$ particle in $C_{0}$, a $B$ or a $C$ particle is allowed in each $C_{i}$. Then the restricted ensembles are given by

$$
\begin{gathered}
\Omega_{A}=\left\{\omega ; \text { there is at least one } A \text {-particle in each } C_{i}\right\}, \\
\Omega_{B, C}=\left\{\omega ; \text { there is at least one } B \text { or } C \text {-particle in each } C_{i}\right\},
\end{gathered}
$$

and

$$
\bar{\Omega}_{C_{\imath}}=\left\{\omega \in \Omega_{C_{i}} ; \text { there is no particle in } C_{i}\right\} .
$$

(3) Diluteness of the Restricted Ensembles. First of all, we define the restricted partition function for the $q^{\text {th }}$ ensemble, $Z_{R}\left(\Lambda \mid \omega^{q}\right)$, with boundary conditions $\omega^{q} \in \Omega_{\bar{\lambda}}^{q}$ :

$$
Z_{R}\left(\Lambda \mid \omega^{q}\right)=\int d v_{\Lambda}\left(\xi \mid \omega^{q}\right) \exp \left(-H\left(\xi \mid \omega^{q}\right)\right) \chi_{\Lambda}^{q}(\xi),
$$

where $\chi_{\Lambda}^{q}$ is the indicator function of $\Omega_{\Lambda}^{q}$. One can define similarly restricted correlation functions.

Let $R$ be the maximal range of all the interactions in $H$ and all the hard-cores in I. Two elementary cubes $C_{i}, C_{j}$ are $R$-close if $d\left(C_{i}, C_{j}\right) \leqq R$, where $d\left(C_{i}, C_{j}\right)$ $=\inf \left\{d(t, s): t \in C_{i}, s \in C_{j}\right\}$ and $d$ is the Euclidean distance. A set of cubes is $R$-connected if any two cubes of that set can be joined by a sequence of $R$-close cubes of that set. 
Now we state our diluteness hypothesis:

(A1) We assume that, for all $\Lambda^{\prime}$ 's that are union of elementary cubes and all $\omega^{q} \in \Omega_{\Lambda}^{q}$, a real valued function $\zeta\left(\cdot \mid \Lambda, \omega^{q}\right)$ is defined on the set of $R$-connected sets of elementary cubes in $\Lambda$, and satisfies the following properties:

$$
\text { i) } Z_{R}\left(\Lambda \mid \omega^{q}\right)=\exp \left(-K|\Lambda|-\sum_{i} K\left(i, \omega^{q}\right)\right)_{\left\{G_{1}, \ldots, G_{n}\right\}} \prod_{i=1}^{*} \zeta\left(G_{i} \mid \Lambda, \omega^{q}\right) \text {, }
$$

where $\sum_{i}$ runs over $i$ 's such that $d\left(C_{i}, \bar{\Lambda}\right) \leqq R$, and $\sum^{*}$ runs over sets such that all $G_{i}$ are $R$-connected and $d\left(G_{i}, G_{j}\right)>R, i \neq j$.

ii) $\zeta\left(G \mid \Lambda, \omega^{q}\right)=1$ if $G$ consists of only one elementary cube.

iii) $\zeta\left(G \mid \Lambda, \omega^{q}\right)$ depends on $\omega^{q}$ only via $\omega_{\Lambda(G, R)}^{q}$, where $\Lambda(G, R)=$ elementary cubes $C$ in $\bar{\Lambda}$ such that $d(C, G) \leqq R\}$. In particular $\zeta\left(G \mid \Lambda, \omega^{q}\right)=\zeta(G)$ does not depend on $\omega^{q}, \Lambda$ if $d(G, \bar{\Lambda})>R$. However, $\zeta(G)$ may depend on $q$. Moreover, $\zeta(G)$ is translation invariant (or periodic) under $\mathbb{Z}^{d}$.

iv) $\sup _{\omega^{q}, A, G}\left|\frac{\zeta\left(G \mid \Lambda, \omega^{q}\right)}{\varepsilon_{0}^{|G|}}\right| \leqq 1$ for some $\varepsilon_{0}$ that will be chosen to be small later. $|G|$ is the number of elementary cubes in $G$.

v) $K$ is independent of $\Lambda, \omega^{q}$, and $K\left(i, \omega^{q}\right)$ depends only on $\omega_{A\left(C_{i}, R\right)}^{q}$; they may both depend on $q$. Moreover, $\sup _{i, \omega^{q}, A}\left|K\left(i, \omega^{q}\right)\right|<\infty$.

Remarks. 1) Formula (2.7) is the general form of most convergent expansions for the partition function: Usually $\varepsilon_{0}$ tends to zero when some parameters (temperature, fugacity) of the system approach their limiting values. Then, for these limiting models around which we perturb, $K$ is the bulk energy and $\sum_{i} K(i, \omega)$ the boundary free energy. We assume in ii) that $\zeta$ (one cube) $=1$ because we can anyway absorb this into $K$.

2) Once we have an expansion of the form (2.7) for the partition function, we obtain the expansion of the free energy from standard algebraic techniques (see Appendix 1). As a result, the limit

$$
f(q)=-\lim _{|\Lambda| \rightarrow \infty} \frac{1}{|\Lambda|} \log Z_{R}\left(\Lambda \mid \omega^{q}\right)
$$

exists, is independent of $\omega^{q}$, and the rate of convergence can be studied in detail.

(4) Peierls' Condition. We start with the definition of contours. Given a configuration $\omega$, a cube $C_{i}$ is regular if $\omega_{C_{i}} \in \Omega_{i}^{q}$ for some $q \in\{1, \ldots, r\}$ and $\omega_{C_{j}} \in \Omega_{j}^{q}$, with the same $q$, for all $j$ such that $d\left(C_{i}, C_{j}\right) \leqq R$. All other cubes are irregular. The set of irregular cubes of $\omega$ is denoted $I(\omega)$. We consider only configurations such that $I(\omega)$ is finite, i.e. $\omega$ belongs to one of the restricted ensembles at infinity. We decompose $I(\omega)$ into maximal $(2 R+1)$-connected components $\Gamma_{1}, \ldots, \Gamma_{n}$. By definition, $d\left(\Gamma_{i}, \Gamma_{j}\right)>2 R+1$ for $i \neq j$. A contour $\left(\Gamma, \omega_{\Gamma}\right)$ is a pair made of such a $(2 R+1)$-connected component, together with the restriction $\omega_{\Gamma}$ of $\omega$, to it. [The somewhat unusual $2 R+1$ constant will be useful later when we show that different contours do not interact too much, e.g. in Eq. (A.14).] $\Gamma$ is called the support of the contour. However, we shall sometimes use the words "the contour $\Gamma$ " instead of "the support of the contour $\left(\Gamma, \omega_{\Gamma}\right)$." There is no ambiguity since $\Gamma$ always denotes the support of a contour. 
The complement of $\Gamma$ is made of several connected components, one of which is infinite. The latter is denoted $\operatorname{Ext} \Gamma$, while $\operatorname{Int} \Gamma$ is the union of all the finite ones. On the boundary of each component the configuration $\omega$ belongs, by definition, to a definite restricted ensemble. We write $o(\Gamma)$ for the restricted ensemble attached to $\operatorname{Ext} \Gamma$, and $\operatorname{Int} \Gamma$ is decomposed into $\bigcup_{m=1}^{r} \operatorname{Int}_{m} \Gamma$ according to the corresponding ensemble. We write

$$
V(\Gamma)=|\operatorname{Int} \Gamma|, \quad \theta(\Gamma)=\Gamma \cup \operatorname{Int} \Gamma, \text { and } \Gamma \subset \Lambda \text { if } d(\Gamma, \bar{\Lambda})>2 R+1 .
$$

We define $Z\left(\Gamma \mid \Lambda, \omega^{q}\right)$ as the partition function obtained by integrating over all configurations in $\Omega_{\Lambda}$ having only one contour $\left(\Gamma, \omega_{\Gamma}\right)$, whose support is $\Gamma$ and having specified restricted ensembles outside of $\Gamma$.

$$
Q\left(\Gamma \mid \Lambda, \omega^{q}\right)=\frac{Z\left(\Gamma \mid \Lambda, \omega^{q}\right)}{Z_{R}\left(\Lambda \mid \omega^{q}\right)} .
$$

\section{Peierls' Condition:}

(A2) We assume that

$$
Q\left(\Gamma \mid \Lambda, \omega^{q}\right) \leqq \exp (-\varrho|\Gamma|)
$$

for all $\Lambda \supset \Gamma, \omega^{q} \in \omega_{\lambda}^{q}$ and all $q=1, \ldots, r$. $\varrho$ will be assumed later to be large.

We also assume that

(ii) there exist $\lambda_{0}>0$ and $c<\infty$ such that

$$
\left\langle\exp \lambda n_{\Gamma}(\cdot)>\left(\Gamma \mid \Lambda, \omega^{q}\right) \leqq \exp (\lambda c \varrho|\Gamma|)\right\rangle \text { for } 0<\lambda<\lambda_{0} .
$$

$n_{\Gamma}(\xi)$ is the total number of particles in $\Gamma$, for the configuration $\xi$ the expectation value $\left\langle>\left(\Gamma \mid \Lambda, \omega^{q}\right)\right.$ is obtained by conditioning on configurations having only one contour, whose support is $\Gamma$ (as in the definition of $Z\left(\Gamma \mid \Lambda, \omega^{q}\right)$ ).

Remark. This is somewhat different from Peierls' condition in the Pirogov-Sinai theory in two respects: First of all, (A2)(i) says that a contour has a higher free energy (not just energy) than the restricted ensembles. Moreover, we shall assume $\varrho$ to be large. But since $\beta$ is absorbed here in the definition (2.4) of $H$, our $\varrho$ corresponds to $\beta \varrho$ in [5], in cases where restricted ensembles reduce to ground state configurations. Part ii) means that contours do not contain too many particles. It will be used later when we perturb $H_{0}$.

(5) Perturbations of the System. We start with a system with a given $H_{0}$, having $r$ restricted ensembles and satisfying (A1)-(A2). Moreover, we assume that the free energies of the restricted ensembles are all equal:

$$
f^{q}\left(H_{0}\right)=f\left(H_{0}\right), \quad q=1,2, \ldots, r .
$$

Now we introduce $r-1$ perturbations, i.e. Hamiltonians $H_{1}, \ldots, H_{r-1}$ like (2.4), with the same range as $H_{0}$ and with norm $\left\|H_{i}\right\|,(2.6)$, equal to one.

We write

$$
H(\mu)=H_{0}+\sum_{i=1}^{r-1} \mu_{i} H_{i}
$$

with $\mu=\left(\mu_{1}, \ldots, \mu_{r-1}\right) \in \mathbb{R}^{r-1}$ and $U(\eta)=\left\{\mu \in \mathbb{R}^{r-1}|| \mu\left|=\max _{i}\right| \mu_{i} \mid \leqq \eta\right\}$. 
We have to assume some smoothness properties for the perturbations:

(A4) The restricted partition functions, defined for the perturbed system $H(\mu)$, have an expansion like (2.7) with coefficients $K(\mu), K\left(i, \omega^{q}, \mu\right)$ and $\zeta(\cdot, \mu)$. Moreover, there exists $\eta>0$, and $C<\infty$ such that, for all $\mu \in U(\eta)$, the derivatives with respect to $\mu,\left|K^{\prime}(\mu)\right|,\left|K^{\prime}\left(i, \omega^{q}, \mu\right)\right|$ are bounded by $C \varrho$ and

$$
\sup \left|\frac{\zeta^{\prime}(G, \mu \mid \Lambda, \omega)}{\varepsilon_{0}^{|G|-2}}\right| \leqq C .
$$

[By (A1) i), $|G| \geqq 2$ if $\zeta \neq 1$.]

From (A4) one concludes that the thermodynamic free energies $f(q, \mu)$ of the restricted ensembles exist and depend smoothly on $\mu$ :

$$
\left|\frac{d}{d \mu_{i}} f(q, \mu)\right| \leqq C^{\prime}(\varrho+1), \quad i=1, \ldots, i-1,
$$

for all $\mu \in U(\eta)$, provided $\varepsilon_{0}$ is small enough (see Appendix 1).

Our last assumption expresses the fact that the perturbations $H_{1}, \ldots, H_{r-1}$, completely lift the degeneracy of the free energies assumed in (A3):

For each $\mu \in U(\eta)$ we define

$$
t(\mu)=(t(1, \mu), \ldots, t(r, \mu)),
$$

where $t(q, \mu)=f(q, \mu)-\min _{1 \leqq k \leqq r} f(k, \mu)$.

$t(\mu) \in O_{r}$, the positive octant in $\mathbb{R}^{r}$.

(A5) $t$ maps $U(\eta)$ into a neighborhood $V$ of the origin in $O_{r}$ and $t^{-1}$ is Lipschitz continuous on $V$ with constant $L$ :

$$
\left|t^{-1}\left(b_{1}\right)-t^{-1}\left(b_{2}\right)\right| \leqq L\left|b_{1}-b_{2}\right|, \quad b_{1}, b_{2} \in V .
$$

This implies that for each subset $A \subset\{1, \ldots, r\}$, there is a $k$-dimensional subset of $U(\eta)$ for which $f(q, \mu)$ reaches its minimum exactly on those $q$ 's that belong to $A$, where $k=r-\# A$.

Now we state our main theorem.

Theorem 6. Assume that we have a system, specified by $H_{0}$ and a set of perturbations $H_{i}, i=1, \ldots, r-1$, satisfying $(A 1)-(A 5)$. Fix all constants except $\varepsilon_{0}, \varrho$, and $\eta$. Assume that $\varepsilon_{0}$ and $\eta$ are small enough and $\varrho$ large enough (depending on $\eta$ ). Then we have a Pirogov-Sinai phase diagram in $U(\eta)$ just as in the Theorem 4, i.e. one point with $r$ phases, $r$ lines with $r-1$ phases etc.

Remark. The relation between $\eta$ and $\varrho$ is discussed in Sect. 4 (Part 3).

\section{Proofs}

The proofs are divided into two parts: In Part A, we prove our main result, Theorem 6. Then in Part B, we prove Theorems 1-5 simply by checking that the hypotheses of Theorem 6 are satisfied in each case. 
A. Proof of Theorem 6. The proof is similar to the one of Pirogov-Sinai [5]. Therefore, we freely refer to their results and we indicate only the main modifications. Moreover, we defer most of the technical parts of the proof to the Appendices.

1. Recursion Relations. We start by showing that the physical model satisfies recursion relations similar to those in Pirogov-Sinai theory. We define the partition function with $q$ boundary conditions as follows: Let $\omega^{q} \in \Omega_{\lambda}^{q}$, and

$$
Z\left(\Lambda \mid \omega^{q}, \mu\right)=\int d v_{\Lambda}\left(\xi \mid \omega^{q}\right) \exp \left(-H_{\mu}\left(\xi \mid \omega^{q}\right)\right) \chi_{\partial \Lambda}^{q}(\xi),
$$

where

$$
\chi_{\partial \Lambda}^{q}(\xi)= \begin{cases}1 & \text { if all contours } \Gamma \text { of } \xi \text { satisfy } d(\Gamma, \bar{\Lambda})>2 R+1, \\ 0 & \text { otherwise }\end{cases}
$$

To each configuration is associated a family of contours and, in each such family, we distinguish the outer contours namely those whose support does not lie in the interior of any other contour of the family. We can evaluate $Z\left(\Lambda \mid \omega^{q}\right)$ (leaving out the index $\mu$ ) by first integrating over all configurations with a given set of outer contours and then over all possible sets of such contours:

$$
Z\left(\Lambda \mid \omega^{q}\right)=\sum_{\partial \subset \Lambda}^{0} \int d v^{*}\left(\omega_{\partial}\right) Z^{0}\left(\omega_{\partial} \mid \Lambda, \omega^{q}\right),
$$

where the sum $\sum^{0}$ runs over all sets $\partial=\left\{\Gamma_{1}, \ldots, \Gamma_{n}\right\}$ of supports of outer contours in $\Lambda$, such that $d\left(\Gamma_{i}, \Gamma_{j}\right)>2 R+1$, for $i \neq j$, and $d(\Gamma, \bar{\Lambda})>2 R+1, \Gamma \in \partial$.

$Z^{0}\left(\omega_{\partial} \mid \Lambda, \omega^{q}, \mu\right)$ is the partition function (3.1), but restricted to those configurations having $\left(\partial, \omega_{\partial}\right)$ as outer contours [leaving out the index $\mu$ in (3.2)]. Finally,

$$
d v^{*}\left(\omega_{\partial}\right)=\prod_{\Gamma \in \partial} d v^{*}\left(\omega_{\Gamma}\right),
$$

and $d v^{*}$ is the restriction of $v$ to contour configurations, namely those configurations $\omega_{\Gamma} \in \Omega_{\Gamma}$ for which all cubes in $\Gamma$ are irregular and which have an extension outside $\Gamma$ for which all cubes $C_{i}$, with $d\left(C_{i}, \Gamma\right) \leqq 1$ are regular. We notice that the support of $d v^{*}$ does not depend on $\omega \in \Omega_{\lambda}^{q}$ because the hard-core has a range at most $R$ and the distance between $\Gamma$ and $\bar{\Lambda}$ is greater than $R$. For the same reason, $d v^{*}\left(\omega_{\partial}\right)$ can be factorized as in (3.3). Also, since the support of the contours are "thick," the components of $\bar{\Gamma}$ decouple if we fix a contour configuration $\omega_{\Gamma}$. Actually, we can write:

$$
Z^{0}\left(\omega_{\partial} \mid \Lambda, \omega^{q}\right)=Z_{R}\left(\Lambda \backslash \theta(\partial) \mid \omega^{q}, \omega_{\partial}^{q}\right) \prod_{\Gamma \in \partial}\left[\exp \left(-H\left(\omega_{\Gamma}\right)\right) \prod_{m=1}^{r} Z\left(\operatorname{Int}_{m} \Gamma \mid \omega_{\Gamma}^{m}\right)\right],
$$

where $\theta(\partial)=\bigcup_{\Gamma \in \partial} \theta(\Gamma)$. In order to justify (3.4) we observe that, since the support of the contours are thick, the exterior of the contours, $\Lambda \backslash \theta(\partial)$ interacts only with that part of the contours which is in the $q^{\text {th }}$ restricted ensemble. We denote this part of the contour configuration by $\omega_{\partial}^{q}$. Therefore, we have a restricted partition function outside $\theta(\partial)$. The same observation applies to $\operatorname{Int} \Gamma$ : For each $m$, we have $m$ boundary conditions on $\operatorname{Int}_{m} \Gamma$ (but there the partition function need not be restricted). Moreover, the support of the contours $\Gamma^{\prime}$ in $\operatorname{Int}_{m} \Gamma$ satisfy

$$
d\left(\Gamma^{\prime}, \overline{\operatorname{Int}_{m} \Gamma}\right)>2 R+1,
$$


because the distance between support of contours satisfies such an inequality. This justifies (3.4).

Now we define interactions between contours, and between a contour and a configuration $\omega \in \Omega_{\lambda}^{q}$. We start with the definition of the proper weight $\psi\left(\omega_{\Gamma}\right)$ of a contour configuration. (This is similar to $\psi(\Gamma)$ in [5].)

Define $Z\left(\omega_{\Gamma} \mid \Lambda, \omega^{q}, \mu\right)$ as the partition function restricted to those configurations whose unique contour is $\left(\Gamma, \omega_{\Gamma}\right)$. [Notice that this is different from $Z^{0}\left(\omega_{\Gamma} \mid \Lambda, \omega^{q}, \mu\right)$ because here $\left(\Gamma, \omega_{\Gamma}\right)$ is the unique outer contour.]

We shall use later the formula, analogous to (3.4) but for this partition function (we drop $\mu$ here):

$$
Z\left(\omega_{\Gamma} \mid \Lambda, \omega^{q}\right)=Z_{R}\left(\Lambda \backslash \theta(\Gamma) \mid \omega^{q}, \omega_{\Gamma}^{q}\right) \exp \left(-H\left(\omega_{\Gamma}\right)\right)\left(\prod_{m=1}^{r} Z_{R}\left(\operatorname{Int}_{m} \Gamma \mid \omega_{\Gamma}^{m}\right)\right) .
$$

Let

$$
\begin{aligned}
\psi\left(\omega_{\Gamma} \mid \Lambda, \omega^{q}, \mu\right)= & -\ln Z\left(\omega_{\Gamma} \mid \Lambda, \omega^{q}, \mu\right)+\ln Z_{R}\left(\Lambda \mid \omega^{q}, \mu\right) \\
& +\sum_{m=1}^{r}(f(q, \mu)-f(m, \mu))\left|\operatorname{Int}_{m} \Gamma\right|
\end{aligned}
$$

Notice that the last term in the right-hand side of (3.6) was added to cancel the volume terms of $\left\{\operatorname{Int}_{m} \Gamma\right\}$ and that they vanish for $\mu=0$.

Peierls' Condition could be stated as follows:

$$
Q\left(\Gamma \mid \Lambda, \omega^{q}\right)=\int d \nu^{*}\left(\omega_{\Gamma}\right) \exp \left[-\psi\left(\omega_{\Gamma} \mid \Lambda, \omega^{q}, \mu=0\right)\right] \leqq \exp (-\varrho|\Gamma|) .
$$

Now $\psi$ allows us to extend Peierls' Condition to $\mu \neq 0$ (part c of the following lemma).

Lemma 1. Under the hypotheses of Theorem 6,

a) $\lim _{\Lambda \rightarrow \infty} \psi\left(\omega_{\Gamma} \mid \Lambda, \omega^{q}, \mu\right)=\psi\left(\omega_{\Gamma} \mid \mu\right)$ exists and is independent of $\omega^{q}$.

b) There exists $c<\infty$ such that $\left|\frac{d}{d \mu_{i}} \psi\left(\omega_{\Gamma} \mid \Lambda, \omega^{q}, \mu\right)\right| \leqq n\left(\omega_{\Gamma}\right)+c \varrho|\Gamma|$. This bound holds uniformly in $\mu \in U(\eta), \omega^{q}, \Lambda, \omega_{\Gamma}$, and $c$ does not depend on $\varrho$.

c) $\forall \mu \in U(\eta)$,

$$
Z(\Gamma, \psi) \equiv \int d v^{*}\left(\omega_{\Gamma}\right) \exp \left(-\psi\left(\omega_{\Gamma} \mid \Lambda, \omega^{q}, \mu\right)\right) \leqq \exp \left(-\frac{\varrho}{2}|\Gamma|\right) .
$$

d) There exists $\lambda>0$ and $c<\infty$ independent of $\varrho$, such that

$$
Z(\Gamma, \psi)^{-1} \int d \nu^{*}\left(\omega_{\Gamma}\right) \exp \left[\lambda n\left(\omega_{\Gamma}\right)-\psi\left(\omega_{\Gamma} \mid \Lambda, \omega^{q}, \mu\right)\right] \leqq \exp (c \varrho|\Gamma|) .
$$

Proof. See Appendix 1.

Now we define the interactions between the contours. First of all, let

$$
\Phi\left(\omega_{\Gamma} \mid \Lambda, \omega^{q}, \mu\right)=\psi\left(\omega_{\Gamma} \mid \Lambda, \omega^{q}, \mu\right)-\psi\left(\omega_{\Gamma} \mid \mu\right)
$$

denote the interaction between the contour $\left(\Gamma, \omega_{\Gamma}\right)$ and the configuration $\omega^{q} \in \Omega_{\lambda}^{q}$. Next, define

$$
\exp \left(-\tilde{W}\left(\omega_{\partial} \mid \Lambda, \omega^{q}\right)\right)=\frac{Z_{R}\left(\Lambda \backslash \theta(\partial) \mid \omega^{q}, \omega_{\partial}^{q}\right)\left(Z_{R}\left(\Lambda \mid \omega^{q}\right)\right)^{|\partial|-1}}{\prod_{\Gamma \in \partial} Z_{R}\left(\Lambda \backslash \theta(\Gamma) \mid \omega^{q}, \omega_{\Gamma}^{q}\right)}
$$


where $|\partial|=\operatorname{card}(\partial)$, and let

$$
W\left(\omega_{\partial} \mid \Lambda, \omega^{q}\right)=\tilde{W}\left(\omega_{\partial} \mid \Lambda, \omega^{q}\right)+\sum_{\Gamma \in \partial} \Phi\left(\omega_{\Gamma} \mid \Lambda, \omega^{q}\right) .
$$

$\Phi$ is a one-body interaction for $\omega_{\Gamma}$ (or it can be viewed as a two-body interaction between $\omega_{\Gamma}$ and $\omega^{q}$ ) while $\tilde{W}$ contains many-body interactions between outer contours (and with $\omega^{q}$ ). Due to assumption (A1), all these interactions are weak (for $\varepsilon_{0}$ small) and rapidly decaying with the distance between outer contours. This will imply that the contour models defined below enjoy all the properties of the one in the Pirogov-Sinai theory (see Appendix 2).

Now we summarize our recursion formulas (3.2) and (3.4):

Define the dilute partition function for $\omega^{q} \in \Omega_{\lambda}^{q}$,

$$
Z_{d}\left(\Lambda \mid \omega^{q}, \mu\right)=Z\left(\Lambda \mid \omega^{q}, \mu\right) / Z_{R}\left(\Lambda \mid \omega^{q}, \mu\right) .
$$

(Dividing by the restricted partition function is the analogue of subtracting the ground state energy in [5].) The crystal partition function for $\omega_{\Gamma}$, where $\omega_{\Gamma}$ is a contour configuration with $o(\Gamma)=q$, is given by

$$
\begin{aligned}
& Z_{c}\left(\omega_{\Gamma} \mid \mu\right) \\
& \quad=\exp \left(-\psi\left(\omega_{\Gamma} \mid \mu\right)\right) \prod_{m=1}^{r}\left[Z_{d}\left(\operatorname{Int}_{m} \Gamma \mid \omega_{\Gamma}^{m}, \mu\right) \exp \left((f(q, \mu)-f(m, \mu))\left|\operatorname{Int}_{m} \Gamma\right|\right)\right] .
\end{aligned}
$$

This can be equivalently defined as

$$
\lim _{\Lambda \rightarrow \infty} Z^{0}\left(\omega_{\Gamma} \mid \Lambda, \omega^{q}, \mu\right) / Z_{R}\left(\Lambda \mid \omega^{q}, \mu\right)
$$

[use (3.4), (3.6), and Lemma 1a) for the existence of the limit].

Lemma 2. Let $\omega^{q} \in \Omega_{\lambda}^{q}$, then

$$
Z_{d}\left(\Lambda \mid \omega^{q}, \mu\right)=\sum_{\partial \subset \Lambda}^{0} \int d v^{*}\left(\omega_{\partial}\right) \exp \left(-W\left(\omega_{\partial} \mid \Lambda, \omega^{q}\right)\right) \prod_{\Lambda \in \partial} Z_{c}\left(\omega_{\Gamma} \mid \mu\right) .
$$

Proof. Insert (3.4) into (3.2) and use the definitions (3.6), (3.9)-(3.11) of $\psi, W, Z_{d}, Z_{c}$.

2. Contour Models. We define now contour models: they do not necessarily correspond to physical models but satisfy recursion relations similar to those of Lemma 2.

Fix $q \in\{1, \ldots, r\}$ and let $\mathscr{C}^{q}$ be the set of contours with $o(\Gamma)=q$. A contour functional $F^{q}$ is a function defined on the set of contour configurations in $\mathscr{C}^{q}$ satisfying:

$$
\sup _{\Gamma, \omega_{\Gamma}} \frac{\left|F^{q}\left(\omega_{\Gamma}\right)\right|}{n\left(\omega_{\Gamma}\right)+|\Gamma|}<\infty .
$$

A contour functional is called a $\tau$-functional if it satisfies the two conditions:

$$
\int d v^{*}\left(\omega_{\Gamma}\right) \exp \left(-F^{q}\left(\omega_{\Gamma}\right)\right) \leqq \exp (-\tau|\Gamma|)
$$

for all contours in $\mathscr{C}^{q}$.

$$
\left\langle\exp \tau^{-1} n(\cdot)\right\rangle\left(\Gamma, F^{q}\right) \leqq \exp \left(\tau^{2}|\Gamma|\right) .
$$

Here $\left\langle>\left(\Gamma, F^{q}\right)\right.$ is the normalized expectation whose normalization is the left-hand side of (3.12). 
Remark. Due to Lemma 1, part c and d, $\psi\left(\omega_{\Gamma} \mid \Lambda, \omega^{q}, \mu\right)$ is a $\tau$-functional with $\tau=\varrho / 2$, for all $\mu \in U(\eta)$ and $\varrho$ large enough $\left[\varrho \geqq \max \left(2 \lambda^{-1}, 4 c\right)\right.$ with $\lambda, c$ as in Lemma 1].

Now we define the crystal partition function with $\tau$-functional $F^{q}$ :

$$
Z\left(\omega_{\Gamma} \mid F^{q}, \mu\right)=\exp \left(-F^{q}\left(\omega_{\Gamma}\right)\right) \prod_{m=1}^{r} Z\left(\operatorname{Int}_{m} \Gamma \mid F^{q}, \mu\right)
$$

where $Z\left(\Lambda \mid F^{q}, \mu\right)$, the dilute partition function, equals:

$$
Z\left(\Lambda \mid F^{q}, \mu\right)=\sum_{\partial \subset \Lambda}^{0} \int d \nu^{*}\left(\omega_{\partial}\right) \exp \left(-W\left(\omega_{\partial}\right)\right) \prod_{\Gamma \in \partial} Z\left(\omega_{\Gamma} \mid F^{q}, \mu\right) .
$$

Here $\Sigma^{0}=$ sum over support of outer contours, and

$$
W\left(\omega_{\partial}\right)=\lim _{\Lambda \rightarrow \infty} W\left(\omega_{\partial} \mid \Lambda, \omega^{q}\right)=\lim _{\Lambda \rightarrow \infty} \tilde{W}\left(\omega_{\partial} \mid \Lambda, \omega^{q}\right)
$$

(the existence of the limit and its independence upon $\omega^{q}$ is proven in Appendix 2). $Z\left(\omega_{\Gamma} \mid F^{q}, \mu\right)$ and $Z\left(\Lambda \mid F^{q}, \mu\right)$ depend explicitly on $\mu$ because $W$ does.

The contour model is defined as the probability distribution for outer contours given by

$$
P\left(\partial \mid F^{q}, \mu\right)=\frac{1}{Z\left(\Lambda \mid F^{q}\right)} \int d \nu^{*}\left(\omega_{\partial}\right) \exp \left(-W\left(\omega_{\partial}\right)\right) \prod_{\Gamma \in \partial} Z\left(\omega_{\Gamma} \mid F^{q}, \mu\right) .
$$

We also define the dilute partition function $Z\left(\Lambda \mid F, \omega^{q}, \mu\right)$ with boundary conditions $\omega^{q} \in \Omega_{\lambda}^{q}$, as

$$
\sum_{\partial \subset \Lambda}^{0} \int d \nu^{*}\left(\omega_{\partial}\right) \exp \left(-W\left(\omega_{\partial} \mid \Lambda, \omega^{q}\right)\right) \prod_{\Gamma \in \partial} Z\left(\omega_{\Gamma} \mid F, \mu\right) .
$$

Now, we can prove, for $\tau$ large enough and $\varepsilon_{0}$ small enough, that these contour models enjoy all the properties of the Pirogov-Sinai contour models. The main difference comes from the interaction between contours and moreover, due to these interactions, the contour models depend explicitly on $\mu$. As we show in Appendix 2, one can prove that

$$
S\left(F^{q}, \mu\right)=\lim _{\Lambda \rightarrow \infty} \frac{1}{|\Lambda|} \log Z\left(\Lambda \mid F^{q}, \omega^{q}, \mu\right)
$$

exists and is independent of $\omega^{q}$. The same limit is obtained with $Z\left(\Lambda \mid F^{q}, \mu\right)$.

Moreover,

$$
\left|S\left(F^{q}, \mu\right)\right| \leqq O\left(e^{-\tau}\right)
$$

and the boundary term

$$
\Delta\left(\Lambda \mid F^{q}, \omega^{q}, \mu\right) \equiv \ln Z\left(\Lambda \mid F^{q}, \omega^{q}, \mu\right)-S\left(F^{q}, \mu\right)|\Lambda|
$$

satisfies

$$
\left|\Delta\left(\Lambda \mid F^{q}, \omega^{q}, \mu\right)\right| \leqq O\left(e^{-\tau}\right)|\partial \Lambda| .
$$

One obtains also the existence of the thermodynamic limit for the correlation functions of the contour model; the latter cluster exponentially and satisfy a Peierls' estimate as in Propositions 2.1 and 2.2 of [5] (see Appendix 2). 
Finally, $S\left(F^{q}, \mu\right)$ depends smoothly on $\mu$ and $F$ : Let $F_{1}, F_{2}$ be two $\tau$ functionals (with the same $q$ ) and $\mu_{1}, \mu_{2} \in U(\eta)$ : Then,

$$
\left|S\left(F_{1}, \mu_{1}\right)-S\left(F_{2}, \mu_{2}\right)\right| \leqq O\left(e^{-\tau}\right)\left(|| F_{1}-F_{2}||+\left|\mu_{1}-\mu_{2}\right|\right),
$$

where

$$
\|F\|=\sup _{\Gamma, \omega_{\Gamma}}\left|\frac{\exp \left(-\frac{\tau}{2} \delta(\Gamma)\right)\left|F\left(\omega_{\Gamma}\right)\right|}{n\left(\omega_{\Gamma}\right)+|\theta(\Gamma)|}\right|
$$

with $\delta(\Gamma)=$ diameter of $\Gamma$. Notice that we have replaced, for convenience, " $a$ " in the definition of the similar norm in [5] by $\tau / 2$ and that $n\left(\omega_{\Gamma}\right)$ has been added.

3. Proof of Theorem 6. We start by defining contour models with a parameter $b \geqq 0$ :

$$
Z(\Lambda \mid F, b, \mu)=\sum_{\partial \subset A}^{0} \exp (b V(\partial)) \int d v^{*}\left(\omega_{\partial}\right) \exp \left(-W\left(\omega_{\partial}\right)\right) \prod_{\Gamma \in \partial} Z\left(\omega_{\Gamma} \mid F\right)
$$

where $V(\partial)=\sum_{\Gamma \in \partial} V(\Gamma)$, and one defines similarly $Z\left(\Lambda \mid F, b, \omega^{q}, \mu\right)$.

The main estimate concerns

$$
\Delta\left(\Lambda \mid F, b, \omega^{q}, \mu\right)=\ln Z\left(\Lambda \mid F, b, \omega^{q}, \mu\right)-S(F, \mu)|\Lambda|-b|\Lambda| .
$$

Note that, by the same proof as the one of Eq. (2.40) in [5], we have

$$
\Delta\left(\Lambda \mid F, b, \omega^{q}, \mu\right) \leqq O\left(e^{-\tau}\right)|\partial \Lambda| .
$$

Lemma 3. Let $F, F^{\prime}$ be two $\tau$ contour functionals (same $q$ ), $\mu, \mu^{\prime} \in U(\eta), b, b^{\prime} \geqq 0$, $\omega, \omega^{\prime} \in \Omega_{\bar{\lambda}}^{q}$ :

$$
\begin{aligned}
& \left|\Delta(\Lambda \mid F, b, \omega, \mu)-\Delta\left(\Lambda \mid F^{\prime}, b^{\prime}, \omega^{\prime}, \mu^{\prime}\right)\right| \\
& \leqq 2\left|b-b^{\prime}\right||\Lambda|+2 \exp \left(-\frac{\tau}{2}+\frac{\tau}{2} \delta(\Lambda)\right)|\Lambda||| F-F^{\prime}|| \\
& \quad+O(1)\left|\mu-\mu^{\prime}\right| \cdot|\Lambda|+O\left(\varepsilon_{0}\right)|\partial \Lambda|
\end{aligned}
$$

Proof. See Appendix 2.

Given these contour models with parameters, one can set a one-to-one correspondence between them and the real model, as in Proposition 2.6 of [5]:

$$
Z_{c}\left(\omega_{\Gamma} \mid \mu\right)=\exp \left(b_{q} V(\Gamma)\right) Z\left(\omega_{\Gamma} \mid F_{\mu}^{q}, \mu\right),
$$

where

$$
b_{q}=f(q, \mu)-S\left(F_{\mu}^{q}, \mu\right)+\alpha,
$$

and $\alpha$ is such that $\inf b_{q}=0$. Moreover, by Lemma 2 and (3.19) we have that

$$
Z_{d}\left(\Lambda \mid \omega^{m}, \mu\right)=Z\left(\Lambda \mid F_{\mu}^{m}, b_{m}, \omega_{m}, \mu\right), \quad \forall m .
$$

$F_{\mu}^{q}$ is given by the equation:

$$
F_{\mu}^{q}\left(\omega_{\Gamma} \mid \mu\right)=\psi\left(\omega_{\Gamma} \mid \mu\right)+T^{q}\left(\omega_{\Gamma} \mid F, \mu, b\right)
$$

where

$$
T^{q}\left(\omega_{\Gamma} \mid F, \mu, b\right)=\sum_{m=1}^{r}\left\{\Delta\left(\operatorname{Int}_{m} \Gamma \mid F^{q}, \mu\right)-\Delta\left(\operatorname{Int}_{m} \Gamma \mid F^{m}, b_{m}, \omega_{\Gamma}^{m}, \mu\right)\right\}
$$


Now we shall show that the right-hand side of (3.23) defines a map from the set of $\tau$ functionals into itself, provided $\tau=\varrho / 4$ and $\varrho$ is large enough: Indeed, (3.12) follows from Lemma 1c) and the estimate

$$
T^{q}\left(\omega_{\Gamma} \mid F, \mu, b\right) \geqq O\left(e^{-\tau}\right)|\Gamma|
$$

[which itself follows from (3.16) and (3.20)], provided

$$
\varrho / 2-O(\exp (-\varrho / 4)) \geqq \varrho / 4
$$

(which holds for $\varrho$ large enough). Moreover, (3.13) can be proven as follows, using the bound

$$
\left|T^{q}\left(\omega_{\Gamma} \mid F, \mu, b\right)-T^{q}\left(\omega_{\Gamma}^{\prime} \mid F, \mu, b\right)\right| \leqq O\left(\varepsilon_{0}\right)|\Gamma|
$$

[which itself follows from the $4^{\text {th }}$ term in the right-hand side of (3.21)]:

$$
\begin{aligned}
\langle\exp (4 n / \varrho)\rangle(\Gamma, \psi+T) & \leqq \exp \left(2 O\left(\varepsilon_{0}\right)|\Gamma|\langle\exp \lambda n\rangle(\Gamma, \psi)\right. \\
& \leqq \exp \left(\left(2 O\left(\varepsilon_{0}\right)+c \varrho\right)|\Gamma|\right)
\end{aligned}
$$

[by Lemma 1d), provided $\varrho \geqq 4 / \lambda]$,

$$
\leqq \exp \left((\varrho / 4)^{2}|\Gamma|\right),
$$

provided $\varrho$ is large enough.

On the other hand, one proves that $T$ is a contraction in the space of $\tau$ functionals equipped with the norm (3.18), just as in [5].

Moreover, we want to know that $F$ is Lipschitz continuous in $\mu$ for the norm $\|F \mid\|$. This follows from (3.23): $\psi$ is Lipschitz continuous by Lemma $1 \mathrm{~b}$ ), and $T$ depends on $\mu$ directly and indirectly (via $F$ and $b$ ). For the direct dependence, one uses (3.21) and for the $F$ and $b$ dependence we follow [5, p. 65]. We know that $f(q, \mu)$ depends smoothly on $\mu$ due to (2.8). One arrives at an estimate

$$
||\left|F_{\mu}^{q}-F_{\mu^{\prime}}^{q}\right| \| \leqq C \varrho\left|\mu-\mu^{\prime}\right|
$$

for some constant $C<\infty$.

Now we use boundary conditions as in [5] to construct the Gibbs states corresponding to the restricted ensembles for which $b_{q}=0$. If $b_{q}=0$ and if we choose $\omega^{q} \in \Omega_{\lambda}^{q}$, then contours will have a small probability in $\Lambda$. Indeed, the distribution of outer contours in the "physical" ensemble [defined by the partition function $Z_{d}$, see (3.10)] is equal to the one in a contour model, with $b=0$, for which a Peierls' estimate holds [see (A.29) in Appendix 2]. This implies that the typical configurations of the thermodynamic limit of the Gibbs states in $\Lambda$ with boundary conditions $\omega^{q}$ will belong to $\Omega_{C_{i}}^{q}$ for most $i$ 's. Thus these Gibbs states will be different for different $q$ 's (such that $b_{q}=0$ ). Moreover, the expansion for contour models outlined in Appendix 2 (which relies on the diluteness of the restricted ensemble) implies exponential clustering of correlation functions and extremality of these Gibbs states in a fairly standard way.

To complete the proof of the main theorem, we still have to show that, for some neighborhood $V$ of 0 in the positive octant $O_{r}$, and for any $b \in V$, one can find a $\mu \in U(\eta)$ such that Eq. (3.22) holds. This is proven almost as in [5], but we have to take into account the non-linear dependence of $f(q, \mu)$ on $\mu$. 
If $S(F)$ were zero, then (3.22) would follow from our assumption (A5). So, define

$$
G(\mu)=t^{-1}\left[b_{q}-S\left(F_{\mu}^{q}, \mu\right)-\min _{1 \leqq q \leqq r}\left(b_{q}-S\left(F_{\mu}^{q}, \mu\right)\right)\right]
$$

with $t$ as in (A5).

If, given $b \in V$, we can find $\mu \in U(\eta)$ so that $\mu=G(\mu)$ then we obtain a set of equations equivalent to (3.22).

Let $a(\mu)=\left(a_{q}(\mu)\right)$, with

$$
a_{q}(\mu)=b_{q}-S\left(F_{\mu}^{q}, \mu\right)-\min _{1 \leqq q \leqq r}\left(b_{q}-S\left(F_{\mu}^{q}, \mu\right)\right) .
$$

Then, since $t^{-1}(0)=0$, and $t^{-1}$ is Lipschitz continuous,

$$
\left|t^{-1}(a(\mu))\right| \leqq L|a(\mu)| \leqq 2 L|b|+2 L\left|S\left(F_{\mu}^{q}, \mu\right)\right| \leqq 2 L|b|+2 L O\left(e^{-\tau}\right)
$$

by (3.16). This shows that $G$ maps $U(\eta)$ into itself if $|b|$ is small enough. To prove that $G$ is a contraction, we write:

$$
\begin{aligned}
\left|t^{-1}(a(\mu))-t^{-1}\left(a\left(\mu^{\prime}\right)\right)\right| & \leqq L\left|a(\mu)-a\left(\mu^{\prime}\right)\right| \leqq 2 L \max _{q}\left|S\left(F_{\mu}^{q}, \mu\right)-S\left(F_{\mu^{\prime}}^{q}, \mu^{\prime}\right)\right| \\
& \leqq O\left(e^{-\tau}\right) L\left(|| F_{\mu}^{q}-F_{\mu^{\prime}}^{q} \||+| \mu-\mu^{\prime} \mid\right)
\end{aligned}
$$

by (3.17),

$$
\leqq O\left(e^{-\tau}\right) L(C \varrho+1)\left|\mu-\mu^{\prime}\right|
$$

by (3.24). This shows that $G$ is a contraction for $\varrho$ large, since $\tau=\varrho / 4$.

B. Proof of Theorems 1-5. As we explained in the beginning of this section, we prove these theorems by checking in each case that the assumptions (A1)-(A5) hold and then using Theorem 6 (or its straightforward extension when we discuss lattice models).

Proof of Theorem 1. We have explained in Sect. II (B, 2, $\left.2^{\circ}\right)$ what the restricted ensembles are in this case: $C_{0}$ contains one lattice site and one restricted ensemble is just the configuration containing an $A$ particle at each site, while the other ensemble is defined by the condition that each site be occupied by a $B$ or a $C$ particle. The restricted partition functions are:

$$
Z_{R}(\Lambda \mid A)=z_{A}^{|A|}, \quad Z_{R}\left(\Lambda \mid \omega^{B C}\right)=z_{0}^{|A|} \sum_{\substack{\omega_{x}=B, C \\ x \in A}} \exp \left(-\beta H_{\Lambda}\left(\omega_{\Lambda} \mid \omega^{B C}\right)\right),
$$

where $\omega^{B C} \in \Omega_{\bar{A}}^{B, C}$ and

$$
H_{\Lambda}\left(\omega_{A} \mid \omega^{B C}\right)=\sum_{X: X \cap A \neq \phi} J_{X}\left(\omega_{X} \mid \omega^{B C}\right) .
$$

The diluteness of the $A$ restricted ensemble is trivial.

We use the standard high temperature expansion to verify the diluteness of the $B-C$ mixture: Write

$$
e^{\beta J_{X}}=\left(e^{\beta J_{X}}-1\right)+1,
$$

and expand the product over $X \cap A \neq \phi$, in (3.25). 
It is obvious that $Z_{R}\left(\Lambda \mid \omega^{B C}\right)$ can be written in the form (2.7) with $\exp (-K)$ $=2 z_{0}, K\left(i, \omega^{B C}\right)=0$. The $G$ 's are ordinary high-temperature graphs, i.e. $R$-connected sets of lattice sites and

$$
\zeta\left(G \mid \Lambda, \omega^{B C}\right)=\left(\frac{1}{2}\right)^{|G|} \sum_{\substack{\omega_{X}=B, C \\ x \in G}} \sum_{\left\{X_{1}, \ldots, X_{k}\right\}} \prod_{i=1}^{k}\left[\exp \left(\beta J_{X_{i}}\left(\omega_{X_{i}} \mid \omega^{B C}\right)\right)-1\right],
$$

where the second sum is over all sets $\left\{X_{1}, \ldots, X_{k}\right\}$ of subsets of $G$ such that $\bigcup_{i=1}^{k} X_{i}=G$

All other conditions in (A1) are easy to check: ii) holds since we assumed in (2.1) that $J_{X}=0$ if $X=$ one site. iii) is obvious from (3.26), with $\zeta(G)$ having the same period as $\left\{J_{X}\right\}$; iv) holds because it is easy to bound (3.26) by $(C \beta)^{k} \leqq(C \beta)^{|G| / R}$ for some suitable constant $C$. We have $k \geqq|G| / R$ because the interaction has a range at most $R$ and $\left\{X_{1}, \ldots, X_{k}\right\}$ covers $G$. Thus, $\varepsilon_{0}=(C \beta)^{1 / R}$. v) is trivial.

In order to check Peierls' condition, we first choose the values of $z_{A}, z_{0}$, and $\beta$ around which we perturb. Using this high-temperature expansion, and the polymer formalism (see Appendix 1) we have, for $\beta$ small,

$$
\ln Z_{R}\left(\Lambda \mid \omega^{B C}\right)=|\Lambda|\left(\ln z_{0}+h(\beta)\right)+\Delta(\beta, \Lambda),
$$

with $|\Delta(\beta, \Lambda)| \leqq g(\beta)|\partial \Lambda|, h(\beta)=\ln 2+O(\beta)$ and $g(\beta) \rightarrow 0$ as $\beta \rightarrow 0$.

If we take

$$
z_{A}=\hat{z}_{A} \equiv z_{0} \operatorname{exph}(\beta)
$$

then the free energies of the restricted ensembles are equal [this verifies (A3)] and, moreover, Peierls' condition is satisfied: due to the hard-core condition, the support of any contour contains a number of empty sets proportional to $|\Gamma|$. Let us check Peierls' condition in the form of inequality (3.7). Inserting (3.5) into (3.6) (for $\mu=0$ ) and using the expansion (3.27) for $\ln Z_{R}\left(\Lambda \mid \omega^{B C}\right)$ with the explicit form of $\Delta(\Lambda, \beta)$ given in Appendix 1, Eq. (A.7) [with $K(i, \omega)=0$ ], one obtains a cancellation of volume factors (outside and inside $\Gamma$ ) leading to a bound:

$$
Q\left(\Gamma \mid \Lambda, \omega^{B C}\right) \leqq \exp \left[-\left(\hat{z}_{A} \#\{\text { empty sites in } \Gamma\}-2 g(\beta)|\Gamma|\right)\right] .
$$

This gives Peierls' condition with $\varrho \cong \hat{z}_{A}$.

Now, since we have two restricted ensembles we need one perturbation: take $z_{A}=\hat{z}_{A} \exp \mu$. For all values of $\mu$, the smoothness hypothesis (A4) is trivial: the $B-C$ restricted partition function does not depend on $\mu$. Now we check (A5): for $\mu>0$,

$$
t(A, \mu)=z_{A}(\exp \mu-1), \quad t(B C, \mu)=0,
$$

and, for $\mu<0$,

$$
t(A, \mu)=0, \quad t(B C, \mu)=z_{A}(1-\exp \mu) .
$$

So, if $b=\left(b_{1}, b_{2}\right) \in O_{2}$,

$$
t^{-1}(b)=\ln \left(1+b_{1} / z_{A}\right)+\ln \left(1-b_{2} / z_{A}\right) .
$$

The Lipschitz continuity of $t^{-1}$ follows from this formula. 
Proof of Theorems 2, 3, 4. The proofs of these theorems are quite similar to each other and to the one of Theorem 1; we discuss in detail the modifications for the proof of Theorem 4; Theorems 2 and 3 are similar. We choose $C_{0}$ small enough so that two particles of different species cannot be found in the same or in adjacent elementary cubes. The restricted ensemble $\Omega_{C_{0}}^{q}$ consist of all admissible configurations in $C_{0}$ containing at least one $q$-type particle. The diluteness of the restricted ensembles follows from the convergence of the Mayer expansion, which holds provided $\beta$ and $r_{0}$ (=the maximal hard-core radius between particles of the same type) are small enough (depending on $z_{q}$, so that $\beta z_{q}$ and $z_{q} r_{0}^{d}$ are small). Note that we perform a Mayer expansion not only for the interactions $\phi_{q q}$ but also for the constraint, imposed by our definition of the restricted ensembles, that there is at least one particle in each cube $C_{i}$. However, for $z_{q}$ large, this is easily handled: the weight of an empty cube in the expansion is $\exp \left(-z_{q}\right)$ (since $\left.\left|C_{i}\right|=1\right)$. The form (2.7) is unusual for a Mayer expansion but one can obtain it by inserting

$$
1=\prod_{\alpha=1}^{n}\left(\sum_{i} I_{C_{i}}\left(x_{\alpha}\right)\right)
$$

into each Mayer graph ( $n$ is the number of particles in the graph, $I_{C_{i}}$ the characteristic function of $C_{i}$ ), and expanding the product. Then one gets a lattice polymer expansion of the form (2.7). Alternatively, one can derive all results using the ordinary form of the expansion and repeating the arguments of part A of this section.

In any case, the Mayer expansion allows us to write:

$$
f\left(q, z_{q}\right)=z_{q}+h_{q}\left(\beta, z_{q}\right), \quad q=0, \ldots, r-1
$$

for the free energies of the restricted ensembles, where $\left|h_{q}\left(\beta, z_{q}\right)\right| \leqq O\left(\delta z_{q}^{2}\right)$, with $\delta=\max \left(\beta, r_{0}^{d}\right)$,

$$
\left|\frac{d}{d z_{q}} h_{q}\left(\beta, z_{q}\right)\right| \leqq O\left(\delta z_{q}\right) .
$$

This implies that, for fixed $z_{0}$ and $\varepsilon$ and for $\delta$ small enough, one can find a point $\left(\hat{z}_{1}, \ldots, \hat{z}_{r-1}\right)$ in $U\left(z_{0}, \varepsilon\right)$ for which all the free energies are equal: $f\left(q, \hat{z}_{q}\right)=f$, $q=0, \ldots, r-1$.

Thus, for this value of $\left(\hat{z}_{q}\right),(\mathrm{A} 3)$ holds and Peierls' condition also holds because - due to the hard-core interactions the number of empty cubes in each contour $\Gamma$ is proportional to $|\Gamma|$,

- the empty cubes imply a loss of free energy of order $\exp [-f \cdot \mid$ empty cubes $\mid]$,

- using the Mayer expansion, one can cancel in (3.6) the volume terms exactly, inside and outside $\Gamma$, leaving only a boundary term $O\left(\delta z_{q}^{2}\right)|\Gamma|$.

Thus, the Peierls' constant $\varrho \cong z_{0}$, since $f \cong z_{0}$ in (3.22), due to (3.30).

Now we take as perturbations of our system $\mu_{1}, \ldots, \mu_{r-1}$ with $\exp \mu_{q}=z_{q} / \hat{z}_{q}$. If $\mu$ is not too large, the Mayer expansion still converges, thus proving (A4).

Note that, with this definition of the perturbations, $U\left(z_{0}, \varepsilon\right)$ in Theorem 4 is, for $\varepsilon$ small enough, included in what we denoted by $U(\eta)$ in the general framework.

Part (ii) of (A2) is trivial for lattice models but not here. Our argument is similar to the proof of Lemma 5.2 in [12]. Write $n_{\Gamma}(\cdot)=\sum_{q=1}^{r} n_{T}^{q}(\cdot)$; it is clearly sufficient to 
bound each

$$
\begin{gathered}
\left\langle\exp \left(\lambda n_{\Gamma}^{q}(\cdot)\right)\right\rangle(\Gamma, \psi), \quad q=1, \ldots, r, \\
\left\langle\exp \left(\lambda n_{\Gamma}^{q}(\cdot)\right)\right\rangle=\int\left\langle\exp \left(\lambda n_{\Gamma}^{q}(\cdot)\right) \mid \underline{y}\right\rangle P(\underline{y}),
\end{gathered}
$$

where in $\langle\mid y\rangle$ we condition on the location $(y)$ of all particles of a species different from $q$. But, since the particles of a different species interact with the $q^{\text {th }}$ species only through hard-core exclusion, $\langle\mid \underline{y}\rangle$ is equivalent to the expectation value in the $q^{\text {th }}$ restricted ensemble in a region $\Lambda_{q}(y)$, where the $q^{\text {th }}$ species is allowed, given $\underline{y}$,

$$
\left\langle\exp \left(\lambda n_{\Gamma}^{q}(\cdot)\right) \mid \underline{y}\right\rangle=\left\langle\exp \left(\lambda n_{\Gamma}^{q}(\cdot)\right)\right\rangle_{R}^{q}\left(\Lambda_{q}(\underline{y})\right) .
$$

Now, since this latter quantity is an expectation value in a restricted ensemble we can use the Mayer expansion to control it. But then, $\langle\exp \lambda n\rangle$ is just a ratio of partition functions with activity $z$ in the denominator and $e^{\lambda} z$ in the numerator.

Thus we can bound this by

$$
\exp \left(O(\lambda) z_{q}\left|\Lambda_{q}(y)\right|\right) \leqq \exp \left(O(\lambda) z_{q}|\Gamma|\right)
$$

but, since $\varrho \cong z_{0}$ and $z_{q} \cong z_{0}$, we have proven (A2)(ii) for small $\lambda$.

Now we come to (A5). In order to obtain $t^{-1}$, we have to find $z_{1}, \ldots, z_{r-1}$ such that, given $b \in O_{r}$

$$
z_{q}-z_{q+1}=b_{q}-b_{q+1}-h_{q}\left(\beta, z_{q}\right)-h_{q+1}\left(\beta, z_{q+1}\right)
$$

for $q=0, \ldots, r-1$ (with $r$ identified to 0 ). By (3.31) the right-hand side of (3.32) is a contraction for $\delta$ small enough. Moreover, the solution is Lipschitz continuous in $b$.

Proof of Theorem 5. Our elementary cube $C_{0}$ will be an elementary cube of the lattice (or a square here since we shall work in $d=2$ for simplicity).

Our restricted ensembles are: $\Omega^{m}, m=1, \ldots, q$, corresponding to a configuration $S_{x}$ equal to $m, \forall x \in \mathbb{Z}^{2}$. These are the ordered ensembles. $\Omega_{i}^{D}$ : all bonds $\langle x y\rangle$ on the boundary of $C_{i}$ are broken, i.e. $S_{x} \neq S_{y}$. This is our disordered ensemble. The diluteness of the ordered ensembles is trivial. For the disordered ensemble, we use a large $q$ expansion, similar to high-temperature expansions $\left(q^{-1}\right.$ replacing $\left.\beta\right)$. The restricted partition function, $Z_{R}\left(\Lambda \mid \omega^{D}\right)$ is just equal to the number of configurations in $\Lambda$ such that $S_{x} \neq S_{y}, \forall\langle x y\rangle \cap \Lambda \neq \emptyset$ (where $S_{y}, y \in \bar{\Lambda}$ is given by $\left.\omega^{D}\right)$. Indeed, for all the disordered configurations, the energy is zero. We write

$$
Z_{R}\left(\Lambda, \omega^{D}\right)=\sum_{\substack{S_{x}=1 \\ x \in \Lambda}}^{q} \prod_{\langle x y\rangle \cap \Lambda \neq \emptyset}\left(1-\delta\left(S_{x}, S_{y}\right)\right), \quad \sum_{S_{x}=1}^{q}=q\left(\sum_{S_{x}=1}^{q} \frac{1}{q}\right) .
$$

Put $K$ in (2.7) equal $-\ln q$, and expand $\Pi\left(1-\delta\left(S_{x}, S_{y}\right)\right)$ in (3.33) into a Mayer series. The result is a sum over sets of two by two disconnected graphs, as in (2.7), with $G=$ connected set of nearest-neighbor pairs

$$
\zeta(G)=\sum_{\substack{S_{x}=1 \\ x \in \underline{G}}}^{q} \frac{1}{q} \prod_{\langle x y\rangle \in G}\left(-\delta\left(S_{x}, S_{y}\right)\right), \quad G=\{x \mid \exists y,\langle x y\rangle \in G\} .
$$

Due to the $\delta$ functions, $\zeta(G)$ is equal to $q^{-(|\underline{G}|-1)}$. But for each $G,|G| \geqq|G| / 2$, $|G|=$ number of n.n. bonds in $G$. So, (A1) is satisfied. 
From (A1) and the expansion for the free energy (Appendix 1) we obtain $f(D, q)$ $=\ln q+O\left(\frac{1}{q}\right)$. On the other hand, $f(m, \beta)$ is a pure energy term, equal to $2 \beta(2$ replaced by $d$ in $\mathbb{Z}^{d}$ ). Thus we can satisfy (A3) by choosing $2 \beta_{0}=f(D, q)$, i.e. $\beta_{0} \cong \frac{1}{2} \ln q$.

As perturbations, we shall only take $\beta-\beta_{0}$ (we could introduce more perturbations, thus getting a larger phase diagram). All the ordered phases are treated as if they were only one phase using symmetry. Given this, (A4) and (A5) are trivial to check.

Now we prove Peierls' estimate (A2). This was proven in [16] using Reflection Positivity. Here we do not use this condition. The proof is somewhat technical but the idea is easy: support of contours contain squares where not all bonds are broken $\left(S_{x} \neq S_{y}\right)$ but not all are unbroken either. These configurations loose on the energy side with respect to the ordered ones (unbroken bonds) and on the entropy side with respect to the disordered ensemble (broken bonds).

Let $Z(\Lambda \mid \underline{u}, \underline{b})$ be the partition function in $\Lambda$ where we sum over all configurations for which the set $\underline{u}$ of unbroken bonds and the set $\underline{b}$ of broken ones are given. Given $\underline{u}$ and $\underline{b}$, let $E_{k}=\{$ sites with $k u$-bonds attached to them $\}$ (we use $E_{k}$ also for $\# E_{k}$ ). For $d=2, k$ runs from 0 to 4 .

We claim that, for any given set $\underline{u}$ and $\underline{b}$ and $\beta=\frac{1}{2} \ln q$,

$$
Z(\Lambda \mid \underline{u}, \underline{b}) \leqq q^{\left(E_{0}+E_{4}\right)} q^{\frac{3}{4}\left(E_{1}+E_{2}+E_{3}\right)} .
$$

Assuming (3.34) we prove Peierls' condition as follows: Define a contour square to be a square $C_{i}$ such that some bonds on its boundary are broken and some are not. With our definition of contours, it is clear that the support of each contour $\Gamma$ contains a number of contour squares proportional to $|\Gamma|$ (since our contours are thick, all squares in $\Gamma$ need not be contour squares). Now, all sites contained in a contour square cannot belong to $E_{0} \cup E_{4}$. Thus, the number of sites in $E_{1} \cup E_{2} \cup E_{3}$ is again proportional to $|\Gamma|$, say larger than $C|\Gamma|$. There are at most $2^{2|\Gamma|}$ ways of specifying which bonds are in $\underline{u}$ and in $\underline{b}$, given $\Gamma$, since they are automatically specified outside $\Gamma$. Finally, the partition function for the restricted ensemble is, when $\beta=\frac{1}{2} \log q$, equal to $q^{|\Gamma|}$ (up to boundary terms), so we get a bound

$$
Q(\Gamma \mid \Lambda, \omega) \leqq 2^{2|\Gamma|} q^{-\frac{1}{4}\left(E_{1}+E_{2}+E_{3}\right)} \leqq 2^{2|\Gamma|} q^{-\frac{C}{4}|\Gamma|},
$$

which implies Peierls' condition. However, one has to add two remarks: we have to use expansions in order to properly control the effect of the boundary conditions on $\Lambda$. Moreover, $\beta_{0}$ is not equal to $\frac{1}{2} \ln q$, but so close to it that it does not affect estimate (3.35).

So we are left with the proof of (3.34). We evaluate the partition function by assigning to each site in $E_{k}$ an energy factor and an entropy factor in such a way that the product of all these factors over all sites gives an upper bound [equal to the right-hand side of (3.34)] on $Z(\Lambda \mid \underline{u}, \underline{b})$.

The energy factors are easy: Put $q^{k / 4}$ for each site in $E_{k}\left(\beta=\frac{1}{2} \ln q\right.$ and each $u$-bonds belongs to two sites). For the entropy factors, we give $q$ to each $E_{0}$ site (since there are no $u$-bonds attached to those sites, the spin has no constraint), $q^{1 / 2}$ 
to each $E_{1}$ and $q^{1 / 4}$ to each $E_{2}$ : Indeed we have to allow for one factor of $q$ for each connected set of $u$ bonds. It is an easy geometrical observation that in each connected set of bonds

$$
1 \leqq \frac{E_{1}}{2}+\frac{E_{2}}{4}
$$

(The simplest cases are: two endpoints, i.e. $E_{1}=2, E_{2}=0$ or a closed loop with at least 4 corners, i.e. $E_{1}=0$ but $E_{2} \geqq 4$.) Multiplying together all these factors gives (3.34).

\section{Alternative Formulation and Concluding Remarks}

In this section, we discuss further the meaning of the hypotheses of our main theorem and how they could be improved to give more general results.

1) We first formulate a more physical hypothesis which would replace (A1) and be sufficient for our purposes. Unfortunately, the proof appears to be more complicated with this hypothesis, so we used (A1). Although assumption (A1) looks rather odd, it expresses, as we have seen in the proof of Theorems 1-5, the general form of most cluster expansions. [We remark that, in general, one cannot avoid, in (2.4), the term $\exp \left(-K|\Lambda|-\sum_{i} K\left(i, \omega^{q}\right)\right)$ : It occurs already in the trivial example where $\Omega^{q}$ is reduced to one point.]

Our alternative hypothesis essentially requires strong clustering properties of the correlation functions in the restricted ensembles. In order to state this clustering property more precisely, let us define $\varrho\left(\omega_{C_{1}}, \ldots, \omega_{C_{n}} \mid \Lambda, \omega^{q}\right)$ as the probability density, in the $q^{\text {th }}$ restricted ensemble [whose partition function is $\left.Z_{R}\left(\Lambda \mid \omega^{q}\right)\right]$, that $\omega_{C_{1}}, \ldots, \omega_{C_{n}}$ are precisely the configurations in the elementary cubes $C_{1}, \ldots, C_{n}$, and the configurations in the other cubes are unspecified.

Let $A_{1}, \ldots, A_{k}$ be sets of elementary cubes and $\omega_{A}=\bigcup_{C \subset A} \omega_{C}$. The partially truncated correlation functions are defined by:

$$
\varrho^{T}\left(\omega_{A_{1}}, \ldots, \omega_{A_{k}}\right)=\sum_{n \geqq 1}(-1)^{n-1}(n-1) ! \sum_{\pi \in P_{n}(\{1, \ldots, k\})} \prod_{\alpha \in \pi} \varrho\left(\omega_{A_{\alpha}}\right),
$$

where $P_{n}$ is the set of partitions of $\{1, \ldots, k\}$ into $n$ elements, $A_{\alpha}=\bigcup_{k \in \alpha} A_{k}$, and we left out the dependence on $\Lambda, \omega^{q}$.

The assumption replacing (A1) can now be stated as follows: There exists $m>0$ such that

$$
\left|\varrho^{T}\left(\omega_{A_{1}}, \ldots, \omega_{A_{k}}\right)\right| \leqq \prod_{i=1}^{k} \varrho\left(\omega_{A_{i}}\right) \exp \left(-m l\left(A_{1}, \ldots, A_{k}\right)\right)
$$

uniformly in $\Lambda, \omega^{q}$, provided $d\left(A_{i}, \bar{\Lambda}\right) \geqq 2 R+1, \forall i$, and $d\left(A_{i}, A_{j}\right) \geqq 2 R+1$ if $i \neq j$. Here $l\left(A_{1}, \ldots, A_{k}\right)$ is the minimal number of elementary cubes in a set $N$ such that $N \cup A_{1} \cup \ldots \cup A_{k}$ is connected (equivalently, it is the tree distance [17] between the $A$ 's). In addition one would replace assumption (A4) by requiring (4.2) to hold for $\mu \in U(\eta)$ with $m$ independent of $\mu$. 
We note that (4.2) can be derived from (A1), and thus holds for the models considered in Theorems 1-5, by using the expansions as in Appendices 1 and 2. Moreover, $m$ is then of order $\left|\ln \varepsilon_{0}\right|$. However, we expect (4.2) to hold quite generally, with some $m>0$, throughout the region in parameter space where the system, in the restricted ensembles, lies in a single phase. This is therefore a much less restrictive hypothesis than (A1). One could weaken this assumption by adding a factor $\prod_{i=1}^{k} c^{\left|A_{i}\right|}$ on the right-hand side of (4.2) at the expense of increasing $\varrho$ in Peierls' condition.

We sketch how (4.2) can be used to replace (A1). One of the main virtues of (A1) is to provide a suitable expansion for $\exp \left(-\tilde{W}\left(\omega_{\hat{\partial}} \mid \Lambda, \omega^{q}\right)\right)$, the interaction between outer contours [see (3.8)]. We can easily relate $\exp (-\widetilde{W})$ to a ratio of correlation functions: In the numerator and the denominator of (3.8) we have partition functions with some boundary condition $\omega^{q}$ in $\theta(\Gamma)$; i.e., the configuration in the elementary cubes of $\theta(\Gamma)$ whose distance from $\Lambda \backslash \theta(\Gamma)$ is less than $R$ belongs to the $q^{\text {th }}$ restricted ensemble. Let $\widetilde{\theta}(\Gamma)$ be the region obtained by deleting these cubes from $\theta(\Gamma)$. Now multiply the numerator and the denominator in (3.8) by

$$
\prod_{\Gamma \in \partial} \exp \left(-H\left(\omega_{T}^{q}\right)\right) Z_{R}\left(\tilde{\theta}(\Gamma) \mid \omega_{T}^{q}\right),
$$

$\omega_{\Gamma}^{q}$ being the configuration in $\theta(\Gamma) \backslash \tilde{\theta}(\Gamma)$.

These transformations show that (leaving out $\omega^{q}, \Lambda$ ):

$$
\exp \left(-\tilde{W}\left(\omega_{\partial}^{q}\right)\right)=\varrho\left(\omega_{\partial}^{q}\right) / \prod_{\Gamma \in \partial \partial} \varrho\left(\omega_{\Gamma}^{q}\right) .
$$

Now, given (4.3), instead of expanding $\exp (-\tilde{W})$ as we do in (A.22), we expand $\varrho\left(\omega_{\partial}^{q}\right)$ in partially truncated correlation functions:

$$
\varrho\left(\omega_{\partial}^{q}\right)=\sum_{\pi \in P(\partial)} \prod_{\left\{\Gamma_{i}\right\} \in \pi} \varrho^{T}\left(\omega_{\Gamma_{1}}^{q}, \ldots, \omega_{\Gamma_{k}}^{q}\right),
$$

where the sum is over all partitions of $\partial$ and the product over all elements of $\pi$. Equation (4.4) follows from the inversion of (4.1).

Now, the reader can check that, if we insert (4.4) in (4.3) and then (4.3) in the partition function of the contour models we obtain a suitable polymer expansion for the latter. Moreover, using (4.2) one shows by techniques similar to the ones of Appendix 2 that this polymer expansion converges.

2) Contrary to (A1), hypothesis (A2)(i) is fairly physical: it requires the presence of a "free energy barrier" between typical configurations of different phases. This condition cannot be relaxed too much, because something has to cause phase separation. However, $\varrho$ presumably needs not be as large as we require it to be, and Dinaburg and Sinai [14] have recently used a weaker Peierls' condition in their analysis of ANNNI models.

3) Condition (A3) simply defines the point in parameter space around which we perturb. One could say, in order to relate this framework to the one of Pirogov and Sinai, that, instead of perturbing around zero temperature, we perturb around any point where there is approximate phase coexistence, i.e. phase coexistence would take place if the phases and the restricted ensembles would coincide. Of course, this is most useful in cases (Potts or Widom-Rowlinson models) where the 
corresponding "low temperature," i.e. $q \rightarrow \infty$ or $z \rightarrow \infty$ limit, is somewhat illdefined. Moreover, by (A5), we have a complete phase diagram in the neighborhood of this point. However, a phase consists not only of one restricted ensemble but also of bubbles, surrounded by contours, of other restricted ensembles: This shifts the point of coexistence of the phases by an amount of order $L e^{-\varrho}$ : the $e^{-\varrho}$ factor comes from the smallest contours and $L$ enters in (A5): $L^{-1}$ is roughly the linear coefficient of the dependence of $f(\mu)$ on $\mu$. Thus we want our set of perturbations to be large enough: Typically $\eta$ should satisfy:

$$
L e^{-\varrho} \ll \eta \ll \varrho .
$$

The upper inequality is necessary so that Peierls' condition [see Lemma 1c)] is not destroyed by the perturbation. Thus, if $\varrho \rightarrow \infty$, we can let $\eta \rightarrow \infty$ provided that (A4), (A5) hold for those values of $\eta$. Of course, (4.5) can be satisfied, for fixed $\eta, L$, by choosing $\varrho$ large but $L, \varrho$, and $\eta$ may be related in a natural way in more general situations, and then condition (4.5) has to be kept in mind.

4) Conditions (A2)(ii) and (A4) are rather technical and could probably be relaxed: (A4) could follow from an expansion in $\mu$ around the $\mu=0$ theory. Part (ii) of $\mathrm{A} 2$ is used to control the derivatives with respect to $\mu$ of $H_{\mu}$ for the expectation inside the contours (for the expectation in the restricted ensemble we can use A4) but it could probably be simplified.

5) Within the context of the Pirogov-Sinai theory, there are two interesting developments. Slawny [8] has given a method, rigorously justified, to compute the asymptotes of the phase diagram and Zahradnik [9], using a different approach to the theory, has proven the uniqueness part of the phase diagram: The phases constructed by the Pirogov-Sinai method are the only ones. We expect that both results can be extended to our situation but we have not checked this. If this is true, one could presumably rigorously justify the asymptotic expansion, in $q^{-1}$, of $\beta(q)$, the transition temperature for the Potts model. Also, our results on the surface tension [18] should be extendable to the present framework (see also [19]).

6) Finally, although we have not considered this case explicitly (it is studied elsewhere $[7,11,13,15])$, it is clear that our method allows an extension of the Pirogov-Sinai theory to continuous spin models, at least for bounded spins. For a class of ferromagnetic models of continuous bounded spins, a low temperature expansion has been given in [20]. We think that the method of that paper could be used here, too; in particular, the extension "around the ground states" in [20] would provide the necessary condition (A1) here. For unbounded spins as well as continuum models not satisfying condition A (see Theorems 2, 3, and 4), we have to generalize our main theorem to a situation where $\|H\|$, defined in (2.6), is no longer finite but where a one-sided bound holds (stability): $H(\xi) \geqq B n(\xi)$.

Several technical complications enter here, e.g. when we estimate derivatives with respect to $\mu$, the boundary terms and so on. However, it does not seem that there is an intrinsic limitation in our method that would prevent us from covering these cases, too.

7) Coming back to our main goal, namely the understanding of the liquid-gas transition in one-component fluids, we shall make two remarks. First of all we consider a Widom-Rowlinson model with two kinds of particles, $A$ and $B$. If there is no interaction between the $B$ particles, we can integrate explicitly over the $B$ 
particles and we are left with a one-component fluid (of $A$ particles) with manybody interactions induced by the $B$ particles [21]. Then the coexistence of $A$ and $B$ particles becomes a liquid( $A$ rich)-gas ( $B$ rich) coexistence. However, in the symmetric case studied by Widom-Rowlinson the $A$ particies were also noninteracting and, consequently, the effective interaction induced by the $B$-particles included infinitely many-body potentials. Here, however, we can allow a small hard-core between the $A$ particles, which implies that we have only finitely many ( $B$ induced) interactions. However, this is still not a very realistic model.

If we consider two body forces, we can only offer the following picture, based on the ideas of this paper: We take elementary cubes whose size is approximately the range of the intermolecular potential. It may be useful to take this range to be large so that we are "close" to the mean-field limit [22]. Define two restricted ensembles, $g$ and $l$, by demanding that the density in each elementary cube be roughly equal to the actual gas density (for $g$ ) or liquid density (for $l$ ).

Now we observe that the free energy, as a function of density, in a box of the size of the intermolecular potential is approximately the van der Waals one: it is minimum for the density being equal to the gas or the liquid one. That is, all other densities "loose" to the gas one for entropy reasons and to the liquid one for energetic reasons. Here we see the similarity with the Potts model. This fact should be responsible for satisfying Peierls' condition (A2).

On the other hand, one can expect that a clustering like (4.2) holds in the gas or the liquid phase. Since the conditions defining the restricted ensembles tend to reduce correlations, (4.2) should hold a fortiori in our restricted ensembles $g$ and $l$. It may even be easier to prove. However, these ideas require a lot of further investigations.

\section{Appendix 1. Diluteness and Restricted Ensembles}

In this appendix, we show how to use the diluteness hypotheses (A1) [and (A4)] in order to prove the existence and smoothness properties (2) of the free energies of the restricted ensembles. We also use this and Peierls' condition in order to prove Lemma 1.

First of all, we apply the polymer or algebraic formalism [23] to (3.7). Let $g$ be the set of $R$-connected sets $G$ in $\mathbb{Z}^{d}$. Let $X: g \rightarrow \mathbb{N}$ be a multiplicity function on $g$; we define $X !=\prod_{G} X(G) !, \operatorname{supp} X=\bigcup_{X(G) \neq 0} G$. We assume that $|\operatorname{supp} X|$ is finite. $X \subset A$ means $\operatorname{supp} X \subset A$, and we write $d(X, E)$ instead of $d(\operatorname{supp} X, E)$ for $E \subset \mathbb{R}^{d}$,

$$
\begin{gathered}
|X|=\sum_{G} X(G)|G|, \\
\alpha(X)=\left\{\begin{array}{lll}
1 & \text { if } X !=1 \text { and if } d\left(G, G^{\prime}\right)>R \text { whenever } X(G), X\left(G^{\prime}\right) \neq 0, \\
0 & \text { otherwise, }
\end{array}\right. \\
\phi(X \mid \Lambda, \omega)=\alpha(X) \prod_{G} \zeta(G \mid \Lambda, \omega)^{X(G)} .
\end{gathered}
$$

Notice that $\phi(X \mid \Lambda, \omega)$ depends in a non-trivial way on $\Lambda, \omega$ only if $d(X, \bar{\Lambda}) \leqq R[$ by (A1)(iii)]. By (2.7) we can write,

$$
Z_{R}\left(\Lambda \mid \omega^{q}\right)=\exp \left(-K|\Lambda|-\sum_{i} K\left(i, \omega^{q}\right)\right) \sum_{X \subset \Lambda} \phi\left(X \mid \Lambda, \omega^{q}\right) .
$$


Now, using the algebraic formalism, we can write:

$$
\sum_{X \subset \Lambda} \phi\left(X \mid \Lambda, \omega^{q}\right)=\exp \sum_{X \subset \Lambda} \phi^{T}\left(X \mid \Lambda, \omega^{q}\right),
$$

where the truncated functions $\phi^{T}$ are defined as usual:

$$
\phi^{T}\left(X \mid \Lambda, \omega^{q}\right)=(X !)^{-1} \prod_{G} \zeta\left(G \mid \Lambda, \omega^{q}\right)^{X(G)} \alpha^{T}(X),
$$

with

$$
\alpha^{T}(X)=\sum_{\gamma \in g(X)}(-1)^{|\gamma|},
$$

where $g(X)$ is a graph whose vertices are all $G$ 's such that $X(G) \neq 0$ (counting multiplicities) and edges are drawn between any two incompatible $G$ 's $\left(d\left(G, G^{\prime}\right) \leqq R\right) ;|\gamma|$ is the number of edges in $\gamma$ and the sum is over all connected subgraphs of $g(X)$.

$\phi^{T}\left(X \mid \Lambda, \omega^{q}\right)$ has the following properties:

- $\phi^{T}\left(X \mid \Lambda, \omega^{q}\right)=\phi^{T}(X)$ is independent of $\Lambda, \omega^{q}$ if $d(X, \bar{\Lambda})>R$,

- $\phi^{T}\left(X \mid \Lambda, \omega^{q}\right)=0$ unless supp $X$ is $R$-connected,

- $\phi^{T}(X)$ has the same periodicity under translations as $\zeta(G)$,

$\sum_{X}^{i} \sup _{\Lambda, \omega^{q}}\left|\phi^{T}\left(X \mid \Lambda, \omega^{q}\right)\right| \varepsilon_{0}^{-|X| / 2} \leqq O\left(\varepsilon_{0}\right)$ for $\varepsilon_{0}$ small enough,

where $\sum_{X}^{i}$ is the sum over all $X$ 's such that the first elementary cube in supp $X$ is $C_{i}$. This estimate (A.3) is essential in all subsequent arguments. It follows in a standard way from the combinatoric estimates:

$$
\sup _{\Lambda, \omega^{q}}\left|\phi^{T}\left(X \mid \Lambda, \omega^{q}\right)\right| \leqq\left(C \varepsilon_{0}\right)^{|X|}
$$

for some $C$ independent of $\varepsilon_{0}$, and the fact that $\phi^{T}(X)=0$ if $\operatorname{supp} X$ is not $R$-connected. (See [23] where somewhat different notations are used.)

Using (A.1), (A.2) we can write

$$
\ln Z_{R}\left(\Lambda \mid \omega^{q}\right)=-K|\Lambda|-\sum_{i} K\left(i, \omega^{q}\right)+\sum_{X \subset \Lambda} \phi^{T}\left(X \mid \Lambda, \omega^{q}\right),
$$

and therefore

$$
f(q, \mu)=-\lim _{\Lambda \rightarrow \mathbb{R}^{d}} \frac{1}{|\Lambda|} \log Z_{R}(\Lambda \mid \omega)
$$

exists, and equals

$$
f(q, \mu)=K(\mu)-\sum_{X}^{0} \phi^{T}(X)
$$

[assuming $\mathbb{Z}^{d}$-invariance of $\zeta(G)$, hence of $\phi^{T}(X)$; otherwise $\Sigma^{0}$ has to be replaced by an average over the period of $\zeta(G)]$.

Moreover, the boundary term

$$
\Delta\left(\Lambda \mid \omega^{q}, \mu\right)=\ln Z_{R}\left(\Lambda \mid \omega^{q}\right)+|\Lambda| f(q, \mu)
$$

is given explicitly by:

$$
-\sum_{i} K\left(i, \omega^{q}\right)+\sum_{\substack{X \subset A \\ d(X, \bar{\Lambda}) \leqq R}}\left(\phi^{T}\left(X \mid \Lambda, \omega^{q}\right)-\phi^{T}(X)\right)-\sum_{C_{i} \subset A} \sum_{X: X \cap \bar{\Lambda} \neq \varnothing}^{i} \phi^{T}(X) .
$$


The second and the third terms in $\Delta\left(\Lambda \mid \omega^{q}, \mu\right)$ are clearly $O\left(\varepsilon_{0}\right)|\partial \Lambda|$, due to (A.3) while the first is $O(1)|\partial \Lambda|$ by assumption (A1)v). Now we give the

Proof of Formula (2.8). Using (A.6) and (A.3) one can easily bound $\left|\frac{d}{d \mu_{i}} f(q, \mu)\right|$ : $\frac{d}{d \mu_{i}} f(q, \mu)=K^{\prime}(q, \mu)-\sum_{X}^{0} \frac{d}{d \mu_{i}}\left(\phi^{T}(X)\right), \quad\left|K^{\prime}(q, \mu)\right| \leqq C \varrho \quad$ by hypothesis (A4),

while

$$
\frac{d}{d \mu_{i}} \phi^{T}(X)=(X !)^{-1} \alpha^{T}(X) \sum_{G}\left(X(G) \zeta^{\prime}(G) \zeta(G)^{X(G)-1} \prod_{G^{\prime} \neq G} \zeta\left(G^{\prime}\right)^{X\left(G^{\prime}\right)}\right.
$$

(leaving out the $\Lambda, \omega^{q}$ dependence).

Using assumption (A4) on $\zeta^{\prime}(G)$ and $\sum_{G}|X(G)| \leqq|X|$, one obtains a bound on $\left|\frac{d}{d \mu_{i}} \phi^{T}(X)\right|$ like (A.4) with $|X|$ replaced by $|X|-2$ (and with a different $C$ ). For small $\varepsilon_{0}$ this implies the convergence of the sum $\sum_{X}^{0}$ in (A.8). However, the latter is not $O\left(\varepsilon_{0}\right)$ but $O(1)$ because the smallest graphs, with $|X|=2$, are $O(1)$. This implies $\left|\frac{d}{d \mu_{i}} f(q, \mu)\right| \leqq C^{\prime}(\varrho+1)$, which is (2.8).

Similarly, one can bound the derivatives with respect to $\mu$ of the boundary term, using (A.7) and (A.4),

$$
\left|\frac{d}{d \mu_{i}} \Delta\left(\Lambda \mid \omega^{q}, \mu\right)\right| \leqq C^{\prime}(\varrho+1)|\partial \Lambda| .
$$

Now we turn to the

Proof of Lemma 1. First of all, we write explicitly $\psi\left(\omega_{\Gamma} \mid \Lambda, \omega^{q}, \mu\right)$, by inserting (3.5) into (3.6) and then using the expansion (A.5) for all the restricted partition functions entering the resulting formula and (A.6) for the corresponding free energies. This gives:

$$
\begin{aligned}
\psi( & \left(\omega_{\Gamma} \mid \Lambda, \omega^{q}, \mu\right) \\
= & H_{\mu}\left(\omega_{\Gamma}\right)+\left(\ln Z_{R}\left(\Lambda \mid \omega^{q}, \mu\right)-\ln Z_{R}\left(\Lambda \backslash \theta(\Gamma) \mid \omega^{q}, \omega_{\Gamma}^{q}, \mu\right)+f(q, \mu)|\operatorname{Int} \Gamma|\right) \\
& -\sum_{m}\left(\ln Z_{R}\left(\operatorname{Int}_{m} \Gamma \mid \omega_{\Gamma}^{m}\right)+f(m, \mu)\left|\operatorname{Int}_{m} \Gamma\right|\right) .
\end{aligned}
$$

It is clear that the terms in the first and second parentheses can be written, using (A.5), as a sum of terms of the form $K\left(i, \omega_{\Gamma}\right)$ for $d\left(C_{i}, \Gamma\right) \leqq R$ and of the form $\phi^{T}\left(X \mid \Lambda, \omega^{q}, \omega_{\Gamma}\right)$ with $d(X, \Gamma) \leqq R$. But then $\phi^{T}\left(X \mid \Lambda, \omega^{q}, \omega_{\Gamma}\right)$ is independent of $\Lambda, \omega^{q}$ unless $|X| \geqq d(\Gamma, \bar{\Lambda})-2 R$. Using (A.4), one sees that $\phi^{T}\left(X \mid \Lambda, \omega^{q}, \omega_{\Gamma}\right)$ with such large $|X|$ 's are exponentially small when $d(\Gamma, \bar{\Lambda}) \rightarrow \infty$. This implies a).

Turning to $b$ ), we can bound the derivatives of the last two parentheses in (A.11) in the same way as we bounded $\frac{d}{d \mu_{i}} \Delta\left(\Lambda \mid \omega^{q}, \mu\right)$ in (A.10). 
Moreover, $\left|\frac{d}{d \mu_{i}} H_{\mu}\left(\omega_{\Gamma}\right)\right|=\left|H_{i}\left(\omega_{\Gamma}\right)\right| \leqq n\left(\omega_{\Gamma}\right)$, since $\left\|H_{i}\right\|=1$. Therefore,

$$
\left|\frac{d}{d \mu_{i}} \psi\left(\omega_{\Gamma} \mid \Lambda, \omega^{q}, \mu\right)\right| \leqq n\left(\omega_{\Gamma}\right)+c \varrho|\Gamma|
$$

for some constant $c$.

This proves b). It also implies c) because we can write

$$
\psi\left(\omega_{\Gamma} \mid \Lambda, \omega^{q}, \mu\right)=\psi\left(\omega_{\Gamma} \mid \Lambda, \omega^{q}, \mu=0\right)+\sum_{i=1}^{r-1} \int_{0}^{\mu_{2}} \frac{d}{d \mu_{i}^{\prime}} \psi\left(\omega_{\Gamma} \mid \Lambda, \omega^{q}, \mu^{\prime}\right) d \mu_{i}^{\prime} .
$$

Now we insert (A.12) into (A.13). We get

$$
\begin{aligned}
& \int d \nu^{*}\left(\omega_{\Gamma}\right) \exp \left(-\psi\left(\omega_{\Gamma} \mid \Lambda, \omega^{q}, \mu\right)\right) \\
& \quad \leqq \exp (\eta(r-1) c \varrho|\Gamma|) \cdot \int d \nu^{*}\left(\omega_{\Gamma}\right) \exp \left(-\psi\left(\omega_{\Gamma} \mid \Lambda, \omega^{q}, \mu=0\right)\right) \exp \left(\eta(r-1) n\left(\omega_{\Gamma}\right)\right) .
\end{aligned}
$$

The first factor is less than $\exp (\varrho / 4|\Gamma|)$ for $\eta$ small enough. We multiply and divide the second factor by

$$
\int d v^{*}\left(\omega_{\Gamma}\right) \exp \left(-\psi\left(\omega_{\Gamma} \mid \Lambda, \omega^{q}, \mu=0\right)\right) .
$$

Then we use Peierls' condition (A2) in the form of Eq. (3.7) and condition (A2)(ii) with $\lambda=\eta(r-1)$ and $\mu=0$ to bound the second factor by $\exp \left(-\frac{3}{4} \varrho|\Gamma|\right)$, again for $\eta$ small enough.

d) is proven similarly.

\section{Appendix 2. Interacting Contour Models}

In this Appendix, we provide a convergent polymer expansion for the contour models, thus proving formulas (3.15)-(3.17). Then we prove Lemma 3 (for contour models with parameters). Our polymer expansion is fairly standard, given that the diluteness hypothesis implies that the interactions between the contours $(W)$ are weak in a suitable sense.

1) Existence of $W\left(\omega_{\partial}\right)$. We start with an explicit formula for $\tilde{W}\left(\omega_{\partial} \mid \Lambda, \omega\right)$ which will also prove (3.14), i.e. the existence of $W\left(\omega_{\partial}\right)$ when the limit $\Lambda \rightarrow \infty$ is taken. Since $W=\Phi+\tilde{W}$, we start with $\tilde{W}$.

By definition

$$
\begin{aligned}
\tilde{W}\left(\omega_{\partial} \mid \Lambda, \omega^{q}\right)= & \sum_{\Gamma \in \partial} \ln Z_{R}\left(\Lambda \backslash \theta(\Gamma) \mid \omega^{q}, \omega_{\Gamma}^{q}\right)-\ln Z_{R}\left(\Lambda \backslash \theta(\partial) \mid \omega^{q}, \omega_{\partial}^{q}\right) \\
& -(|\partial|-1) \ln Z_{R}\left(\Lambda \mid \omega^{q}\right),
\end{aligned}
$$

with $|\partial|=\operatorname{card}(\partial)$.

Inserting (A.5) into the above formula, gives

$$
\begin{aligned}
\tilde{W}\left(\omega_{\partial} \mid \Lambda, \omega^{q}\right)= & \sum_{X \subset \Lambda}\left(\sum_{\Gamma \in \partial} \phi^{T}\left(X \mid \Lambda, \omega^{q}, \omega_{\Gamma}^{q}\right) n(X, \Gamma)\right) \\
& -n(X, \partial) \phi^{T}\left(X \mid \Lambda, \omega^{q}, \omega_{\partial}^{q}\right)-(|\partial|-1) \phi^{T}\left(X \mid \Lambda, \omega^{q}\right),
\end{aligned}
$$


where

$$
n(X, \partial)= \begin{cases}1 & \text { if } X \subset \Lambda \backslash \theta(\partial) \\ 0 & \text { otherwise }\end{cases}
$$

[so that $n(X, \partial)=0$ whenever $\phi^{T}\left(X \mid \Lambda, \omega^{q}, \omega_{\partial}^{q}\right)$ is not well defined]. Indeed, we observe that in (A.14) all the terms proportional to $K$ or of the form $\sum_{i} K(i, \omega)$ cancel because, by definition, $d\left(\Gamma, \Gamma^{\prime}\right)>2 R+1$ for $\Gamma, \Gamma^{\prime} \in \partial$ and $d(\Gamma, \bar{\Lambda})>2 R+1$, too. So we have only $\phi^{T}(X)$ terms. Moreover, by inspection of (A.15) one sees that only those $X^{\prime}$ 's satisfying $d(X, \Gamma) \leqq R$, and $d\left(X, \Gamma^{\prime}\right) \leqq R$ for at least two contours $\Gamma, \Gamma^{\prime} \in \partial$ give a non-zero contribution. Indeed, if $d(\Gamma, X)>R$, then, by assumption (A1)(iii) $\phi^{T}\left(X \mid \Lambda, \omega^{q}, \omega_{\partial}^{q}\right)$ does not depend on $\omega_{\Gamma}^{q}$.

A similar formula can be derived for $\Phi\left(\omega_{\Gamma} \mid \Lambda, \omega^{q}\right)$, where the only $X$ 's that can contribute to $\Phi$ satisfy $d(X, \Gamma) \leqq R$, and $d(X, \bar{\Lambda}) \leqq R$ [as we noticed in the proof of Lemma 1a), Appendix 1].

From (A.15) and the exponential decay of $\phi^{T}(X)$ with $|X|$ [see Eq. (A.4)], we get

$$
\lim _{\Lambda \rightarrow \infty} \tilde{W}\left(\omega_{\partial} \mid \Lambda, \omega^{q}\right)=\tilde{W}\left(\omega_{\partial}\right)
$$

and its independence upon $\omega^{q}$. Actually, one has a bound of the form:

with $M \sim O\left(\left|\ln \varepsilon_{0}\right|\right)$, and

$$
\left|\tilde{W}\left(\omega_{\partial} \mid \Lambda, \omega^{q}\right)-\tilde{W}\left(\omega_{\partial}\right)\right| \leqq \sum_{\Gamma \in \partial}|\Gamma| \exp (-M d(\Gamma, \bar{\Lambda}))
$$

$$
\left|\Phi\left(\omega_{\Gamma} \mid \Lambda, \omega^{q}\right)\right| \leqq|\Gamma| \exp (-M d(\Gamma, \bar{\Lambda})) .
$$

Thus $\Phi \rightarrow 0$ and $W\left(\omega_{\partial}\right)=\tilde{W}\left(\omega_{\partial}\right)$.

Moreover, one can estimate the interaction between one contour and any set of other contours as follows: Let $\Gamma \in \partial$ and $\partial^{\prime}=\partial \backslash\{\Gamma\}$, then,

$$
\sup _{\omega_{\partial}}\left|W\left(\omega_{\partial}\right)-W\left(\omega_{\partial^{\prime}}\right)\right| \leqq O\left(\varepsilon_{0}\right)|\Gamma| .
$$

This again follows easily from the bound (A.4) on the $\phi^{T}(X)$ and the fact that the left-hand side of (A.16) is bounded from above by $\sup _{\omega_{\partial}} \sum_{X: d(X, \Gamma) \leqq R}\left|\phi^{T}\left(X \mid \Lambda, \omega_{\partial}\right)\right|$.

Although (A.16) is only used explicitly in the proof of the Peierls' estimate [see (A.29) below] it is the basic bound that allows us to prove the convergence of our polymer expansion: the interaction between a contour and the other contours is bounded by $|\Gamma|$ times a small coefficient. But, by the definition of $\tau$ functionals, every contour has a weight $\exp (-\tau|\Gamma|)$ which controls the $O(|\Gamma|)$ divergence in (A.16).

2) Polymer Expansion for Contour Models. Now we give the polymer expansion of $Z(\Lambda \mid F)$. $\left[Z\left(\Lambda \mid F, \omega^{q}\right)\right.$ can be treated in a similar way and the independence of $S(F)$ on $\omega^{q}$ follows easily from this expansion.] Writing explicitly the inductive definition of $Z(\Lambda \mid F)$, one gets:

$$
\begin{aligned}
Z(\Lambda \mid F) & =\sum_{\partial \subset \Lambda}^{0} \int d \nu^{*}\left(\omega_{\partial}\right) \exp \left(-W\left(\omega_{\partial}\right)\right) \prod_{\Gamma \in \partial} Z\left(\omega_{\Gamma} \mid F\right) \\
& =\sum_{\partial \subset \Lambda} \int d \nu^{*}\left(\omega_{\partial}\right) \exp \left(-\bar{W}\left(\omega_{\partial}\right)\right) \prod_{\Gamma \in \partial} \exp \left(-F\left(\omega_{\Gamma}\right)\right),
\end{aligned}
$$

where the first equality is just the definition. 
In the second equality, the sum runs over all families $\partial$ of contours with $o(\Gamma)=q$ for $\Gamma \in \partial, d\left(\Gamma, \Gamma^{\prime}\right) \geqq 2 R+1$ for $\Gamma, \Gamma^{\prime} \in \partial$, and not just over outer contours. $\bar{W}\left(\omega_{\partial}\right)$ is defined as follows: we say that a contour $\Gamma$ separates a set of contours $\partial$ if there exist two contours $\Gamma_{1}, \Gamma_{2}$ in $\partial$ that lie in different connected components of $\bar{\Gamma}$. We say that $\Gamma$ surrounds $\partial$ if $\partial$ lies inside one connected component of Int $\Gamma$. Finally, we say that a subset $\partial^{\prime} C \partial$ is a set of outer contours relative to $\partial$ if there is no contour $\Gamma \in \partial$ that separates $\partial^{\prime}$, no contour $\Gamma \in \partial^{\prime}$ that surrounds $\partial^{\prime} \backslash\{\Gamma\}$, and if $\partial^{\prime}$ cannot be enlarged while maintaining the first two properties (notice that a contour $\Gamma \in \partial$ may surround $\partial$ ). Then

$$
\bar{W}\left(\omega_{\partial}\right)=\sum_{\partial^{\prime} \subset \partial} W\left(\omega_{\partial^{\prime}}\right),
$$

where the sum runs over all subsets $\partial^{\prime} \subset \partial$ that are outer relative to $\partial$.

Now, using $W\left(\omega_{\partial}\right)=\tilde{W}\left(\omega_{\partial}\right)$ and inserting (A.15) into (A.18), we obtain,

$$
\bar{W}\left(\omega_{\partial}\right)=\sum_{X} \sum_{\partial^{\prime} \subset \partial}\left\{\sum_{\Gamma \in \partial^{\prime}} \phi^{T}\left(X \mid \omega_{\Gamma}^{q}\right) n(X, \Gamma)-\phi^{T}\left(X \mid \omega\left(\partial^{\prime}(X)\right)\right) n\left(X, \partial^{\prime}\right)-\left(\left|\partial^{\prime}\right|-1\right) \phi^{T}(X)\right\},
$$

where

$$
\omega(\partial(X))=\left\{\omega_{\Gamma} \mid \Gamma \in \partial(X)\right\} \quad \text { and } \quad \partial(X)=\{\Gamma \in \partial \mid d(\Gamma, X) \leqq R\}
$$

[similarly for $\left.\partial^{\prime}(X)\right]$. So $\partial(X)$ are the $\Gamma$ 's in $\partial$ on which $\phi^{T}\left(X \mid \omega_{\partial}\right)$ can depend. We abbreviate:

$$
\bar{W}\left(\omega_{\partial}\right)=\sum_{X} \psi(X \mid \omega(\partial(X)),
$$

where $\psi(X \mid \omega(\partial(X))$ is defined through (A.19). Obviously, $\psi(X \mid \omega(\partial(X))$ depends only on $\omega(\partial(X))$ and equals zero unless $\operatorname{supp} X$ is $R$-connected and card $\partial(X)>2$. Moreover,

$$
|\psi(X \mid \omega(\partial(X)))| \leqq\left(C \varepsilon_{0}\right)^{|X|}
$$

for some constant $C$ independent of $\varepsilon_{0}$ [different from the one in (A.4)]. Indeed, each $\phi^{T}(X)$ entering into the definition of $\psi$ satisfies such a bound; moreover, the number of $\partial^{\prime}$ that give a non-zero contribution is at most $O(|X|)$ (because each $\partial^{\prime}$ is outer relative to $\partial$ ) and the number of $\Gamma$ 's in $\partial^{\prime}$ giving a contribution that is not cancelled by one $\phi^{T}(X)$ is $O(|X|)$. These factors can be absorbed into $C$ in (A.21).

Now we insert (A.20) into (A.17) and expand:

$$
\exp (-\bar{W})=\prod_{X}(\exp (-\psi(X))-1+1)=\sum_{\left\{X_{1}, \ldots, X_{m}\right\}} \prod_{i=1}^{m}\left(\exp \left(-\psi\left(X_{i}\right)\right)-1\right),
$$

where we write $\psi(X)$ as an abbreviation for $\psi(X \mid \omega(\partial(X))$.

Combining (A.22) and (A.17) we obtain:

$$
\begin{aligned}
Z(\Lambda \mid F)= & \sum_{\partial \subset \Lambda} \sum_{\left\{X_{1}, \ldots, X_{m}\right\}} \int d v^{*}\left(\omega_{\partial}\right) \exp \left(-F\left(\omega_{\partial}\right)\right) \\
& \cdot \prod_{i=1}^{m}\left(\exp \left(-\psi\left(X_{i} \mid \omega\left(\partial\left(X_{i}\right)\right)\right)-1\right)\right.
\end{aligned}
$$

with $F\left(\omega_{\partial}\right)=\sum_{\Gamma \in \partial} F\left(\omega_{\Gamma}\right)$. 
Define $\underline{\partial}=\bigcup_{\Gamma \in \partial} \Gamma$ and, for each term in (A.23), decompose

$$
\underline{\partial} \cup\left(\bigcup_{i=1}^{m} \operatorname{supp} X_{i}\right)=P_{1} \cup \ldots \cup P_{n}
$$

into maximal $R$-connected subsets. Thus $d\left(P_{i}, P_{j}\right)>R$ if $i \neq j$. The integral in (A.23) factorizes over the contribution from the different $P_{i}$ 's: if $\operatorname{supp} X \subset P_{i}$, then $\psi(X \mid \omega(\partial(X)))$ cannot depend on $\omega_{\Gamma}$ for $\Gamma \subset P_{j}, j \neq i$, because $d\left(P_{i}, P_{j}\right)$ is larger than $R$ and therefore, $d(X, \Gamma)>R$, which implies $\Gamma \notin \partial(X)$.

The $P_{i}$ 's will be our (new) polymers. The activity $\xi(P)$ of a polymer $P$ is:

$$
\xi(P)=\sum^{P} \int d v^{*}\left(\omega_{\partial}\right) \exp \left(-F\left(\omega_{\partial}\right)\right) \prod_{i=1}^{m}\left(\exp \left[-\psi\left(X_{i} \mid \omega\left(\partial\left(X_{i}\right)\right)\right)\right]-1\right)
$$

where the sum $\sum^{P}$ runs over all $\partial$ 's and all sets $\left\{X_{1}, \ldots, X_{m}\right\}$ of multiplicity functions such that

$$
P=\underline{\partial} \cup\left(\bigcup_{i=1}^{m} \operatorname{supp} X_{i}\right)
$$

If $P=\emptyset, \xi(P)=1$ and if $m=0$ (empty set of multiplicity functions) in (A.24), $\prod_{i=1}^{0}=1$.

We may rewrite (A.23):

$$
Z(\Lambda \mid F)=\sum_{\left\{P_{1}, \ldots, P_{n}\right\}} \prod_{i=1}^{n} \xi\left(P_{i}\right)
$$

where the sum runs over all sets of two by two "disjoint" polymers $[P$ disjoint from $P^{\prime}$ means $\left.d\left(P, P^{\prime}\right)>R\right]$.

We can estimate $|\xi(P)|$ as follows: Using $\left|e^{z}-1\right| \leqq|z| e^{|z|}$, we have

$$
|\xi(P)| \leqq \Sigma^{P} \int d v^{*}\left(\omega_{\partial}\right) \exp \left(-F\left(\omega_{\partial}\right)\right) \prod_{i=1}^{m}\left|\psi\left(X_{i}\right)\right| \exp \left(\sum_{i=1}^{m}\left|\psi\left(X_{i}\right)\right|\right) .
$$

Using (A.21) and the fact that $\psi(X \mid \omega(\partial(X)))=0$ unless card $\partial(X) \geqq 2$, we obtain

$$
\sum_{i=1}^{m}\left|\psi\left(X_{i}\right)\right| \leqq O\left(\varepsilon_{0}\right) \sum_{\Gamma \in \partial}|\Gamma|
$$

Indeed, each $X_{i}$ has to be close to some $\Gamma \in \partial$ if $\psi(X) \neq 0$. [Note that this bound is analogous to (A.16).]

Moreover, using (A.21) again,

$$
\prod_{i=1}^{m}\left|\psi\left(X_{i}\right)\right| \leqq \exp \left(-M \sum_{i=1}^{m}\left|X_{i}\right|\right)
$$

where $M=O\left(\left|\ln \varepsilon_{0}\right|\right)$.

Finally, since $F$ is a $\tau$-functional.

$$
\int d v^{*}\left(\omega_{\partial}\right) \exp \left(-F\left(\omega_{\partial}\right)\right) \leqq \exp \left(-\tau \sum_{\Gamma \in \partial}|\Gamma|\right) .
$$


Inserting all these estimates into (A.26), we obtain

$$
|\xi(P)| \leqq \sum^{P} \exp \left(-M \sum_{i=1}^{n}\left|X_{i}\right|\right) \exp \left(-\left(\tau-O\left(\varepsilon_{0}\right)\right) \sum_{\Gamma \in \partial}|\Gamma|\right)
$$

Since $P$ is $R$-connected the sum over $\partial$ 's and over sets of multiplicity functions can be controlled in a standard way, leading to our final bound:

$$
|\xi(P)| \leqq \exp (-C|P|)
$$

where $|P|=\operatorname{card}\left(\left\{i \in \mathbb{Z}^{d} \mid C_{i} \subset P\right\}\right)$ and $C=O\left(\min \left(M, \tau-O\left(\varepsilon_{0}\right)\right)\right)$ goes to $\infty$ when $\tau, \varepsilon_{0}^{-1} \rightarrow \infty$.

Given (A.25) and (A.28) one can obtain, from the algebraic formalism, a convergent expansion for $\ln Z(\Lambda \mid F)$, as we did in Appendix 1 for the free energy of the restricted ensembles.

If we include the $\omega$-dependence in $Z(\Lambda \mid F, \omega)$, then one obtains a formula similar to (A.26) but the activity of the polymers close to $\bar{\Lambda}$ are modified. The existence of $S(F)$ is proven just as (A.6) in Appendix 1. In order to prove (3.15) we observe that, if $P \neq \emptyset$, then for each non-zero term of the sum $\sum^{P}$ in (A.24), $\partial \neq \emptyset$ : indeed, $\psi(X \mid \omega(\partial(X)))=0$ if $\partial(X)=\emptyset$ and $\partial(X) \subset \partial$. This and (A.27) implies that $|\xi(P)| \leqq O\left(e^{-\tau}\right)$. Since $S(F)$ is given by a convergent series of truncated functions of polymers, this last bound can be easily extended to $S(F)$.

From this expansion, one also gets, in a standard fashion, the bound (3.16) on the boundary term $\Delta(\Lambda \mid F, \omega, \mu)$, and the existence and clustering of the correlation functions. We could get Peierls' estimate from the expansion, but we prefer to do it as follows: Write

$$
P(\Gamma)=Z(\Lambda \mid F)^{-1} \sum_{\partial: \Gamma \in \hat{\partial}} \int d \nu^{*}\left(\omega_{\partial}\right) \exp (-\bar{W}(\omega)) \prod_{\Gamma \in \hat{\partial}} \exp (-F(\omega))
$$

Let $\partial^{\prime}=\partial \backslash\{\Gamma\}$ and bound $P(\Gamma)$ from above by restricting the sum in $Z(\Lambda \mid F)$ to the sets $\partial^{\prime}$. Now use (A.16) (which extends easily to $\bar{W}$ ) in the numerator to compare it with the denominator; we obtain

$$
P(\Gamma) \leqq \exp \left(\left(O\left(\varepsilon_{0}\right)-\tau\right)(\Gamma)\right),
$$

which is Peierls' estimate.

Now we prove (3.17). It is enough to prove:

$$
\left|\frac{d}{d \mu} \log Z(\Lambda \mid F, \mu)\right| \leqq|\Lambda| O\left(e^{-\tau}\right)
$$

and

$$
\left|\frac{d}{d t} \log Z\left(\Lambda \mid F_{t}, \mu\right)\right| \leqq|\Lambda| O\left(e^{-\tau}\right)|| F_{1}-F_{2} \mid \|,
$$

with $F_{t}=t F_{1}+(1-t) F_{2}$.

Using (A.17) we write

$$
\left|\frac{d}{d \mu} \log Z(\Lambda \mid F, \mu)\right|=\left|\left\langle\frac{d \bar{W}}{d \mu}(\cdot)\right\rangle\right|,
$$


where \langle\rangle is defined in the obvious way by (A.17). Observing that, in the definition of $\bar{W}$,(A.19), the only $X$ 's that contribute must satisfy $d(\Gamma, X) \leqq R$ for at least one (in fact, two) $\Gamma$ 's in $\partial$, and using the bounds (A.4) on $\phi^{T}$ and its derivative with respect to $\mu$, we easily get:

$$
\left|\frac{d}{d \mu} \bar{W}\left(\omega_{\partial}\right)\right| \leqq O(1) \sum_{\Gamma \in \partial}|\Gamma| .
$$

Thus the right-hand side of (A.32) is bounded by

$$
O(1) \sum_{\partial \subset A} P(\partial) \sum_{\Gamma \in \partial}|\Gamma|=O(1) \sum_{\Gamma \subset A} P(\Gamma)|\Gamma| .
$$

[Here $P(\partial)$ denotes one of the terms in the sum (A.28).] By Peierls' estimate, (A.29), this last quantity is less than $O\left(e^{-\tau}\right)|\Lambda|$, which proves (A.30).

Now we prove (A.31). By a calculation similar to the one above and the definition (3.18) of $\|F\|$, we get

$$
\left|\frac{d}{d t} \log Z\left(\Lambda \mid F_{t}, \mu\right)\right| \leqq\left\|F_{1}-F_{2}\right\| \cdot \cdot\left(\sum_{\Gamma \subset A} P(\Gamma) e^{\tau \delta(\Gamma) / 2}\left(|\theta(\Gamma)|+\left\langle n\left(\omega_{\Gamma}\right)\right\rangle_{\Gamma}\right)\right),
$$

where \langle\rangle$_{\Gamma}$ is just $\langle>$ as in (A.32) conditioned on $\Gamma$. Now, notice that, by convexity, we need only to bound the derivative $\frac{d}{d t}$ for $t=0$ and 1 . But, in these cases, $F_{t}=F_{1}$ or $F_{2}$ is a $\tau$-functional and we can use (3.13) to prove:

$$
\left\langle n\left(\omega_{\Gamma}\right)\right\rangle_{\Gamma} \leqq O\left(\tau^{3}\right)|\Gamma| \exp \left(O\left(\varepsilon_{0}\right)|\Gamma|\right) .
$$

To prove (A.34) we use (A.16) in the numerator and the denominator of \langle\rangle$_{T}$ in order to "decouple" $\Gamma$ from the other contours. Thus we have

$$
\langle n\rangle_{\Gamma} \leqq \exp \left(O\left(\varepsilon_{0}\right)|\Gamma|\right)\langle n\rangle(\Gamma, F)
$$

which, by (3.13), implies (A.34).

Now, inserting (A.34) into (A.33) and using Peierls' estimate (A.29), we easily obtain (A.31) for $\tau$ large enough.

We remark that we could have bounded the derivatives (A.30) and (A.31) (for $t=0$ or 1 ) using directly the polymer expansion as we did in (A.8), (A.9). However, these more "probabilistic" arguments (i.e. dependent on $F, \bar{W}$ being real) are useful for our last estimate:

Proof of Lemma 3. This is the analogue of Proposition 2.5 in [5]. The dependence upon $b$ is treated just as in [5]. Using (3.19), we estimate

$$
\begin{aligned}
& \left|\frac{d}{d \mu} \log Z(\Lambda \mid F, b, \mu)\right| \leqq Z^{-1} \sum_{\partial \subset \Lambda}^{0} e^{b V(\partial)} P(\partial) \\
& \quad \cdot\left(\left|\left\langle\frac{d}{d \mu} W(\cdot)\right\rangle(\partial)\right|+\sum_{\Gamma \in \partial} \sum_{m=1}^{r} \sup _{\omega_{\Gamma}^{m}}\left|\left\langle\frac{d}{d \mu} \bar{W}(\cdot)\right\rangle\left(\operatorname{Int}_{m} \Gamma, \omega_{\Gamma}^{m}\right)\right|\right),
\end{aligned}
$$

where $P(\partial)=\int d v^{*}\left(\omega_{\partial}\right) \exp \left(-W\left(\omega_{\partial}\right)\right) \prod_{\Gamma \in \partial} Z\left(\omega_{\Gamma} \mid F\right)$ and \langle\rangle$(\partial)$ is the expectation conditioned on $\partial$ being the set of outer contours; \langle\rangle$\left(\operatorname{Int}_{m} \Gamma, \omega_{\Gamma}^{m}\right)$ is defined as in 
(A.17) with $\Lambda=\operatorname{Int}_{m} \Gamma$ and b.c. $\omega_{\Gamma}^{m}$. Clearly, with the bounds that we have on $\frac{d}{d \mu} W$,

$$
\left|\left\langle\frac{d}{d \mu} W(\cdot)\right\rangle(\partial)\right| \leqq O(1) \sum_{\Gamma \in \partial}|\Gamma|
$$

and

$$
\sup _{\omega_{\Gamma}^{m}}\left|\left\langle\frac{d}{d \mu} \bar{W}(\cdot)\right\rangle\left(\operatorname{Int}_{m} \Gamma, \omega_{\Gamma}^{m}\right)\right| \leqq O(1)\left|\operatorname{Int}_{m} \Gamma\right| .
$$

Since, for each set $\partial$ of outer contours in $\Lambda, \sum_{\Gamma \in \partial}|\Gamma|+V(\partial) \leqq|\Lambda|$ and $Z^{-1} \sum_{\partial \subset \Lambda}^{0} e^{b V(\partial)} P(\partial)=1$, we have the bound

$$
\left|\frac{d}{d \mu} \log Z(\Lambda \mid F, b, \mu)\right| \leqq O(1)|\Lambda|
$$

which yields the third part of (3.21) ( since we have already a bound on $\frac{d}{d \mu} S(F, \mu)$ by (A.30)). The fourth term in (3.21) follows from:

$$
\sup _{\omega_{\partial}}\left|W\left(\omega_{\partial} \mid \Lambda, \omega\right)-W\left(\omega_{\partial} \mid \Lambda, \omega^{\prime}\right)\right| \leqq O\left(\varepsilon_{0}\right)|\partial \Lambda|, \quad \forall \omega, \omega^{\prime} \in \Omega_{\lambda}^{q},
$$

which itself can be easily derived from (A.15) and properties of $\phi^{T}(X)$ 's and implies

$$
\left|\log \frac{Z(\Lambda \mid F, b, \omega, \mu)}{Z\left(\Lambda \mid F, b, \omega^{\prime}, \mu\right)}\right| \leqq O\left(\varepsilon_{0}\right)|\partial \Lambda| \text {. }
$$

Now we control the $F$-dependence in (3.21). Let $F_{t}=t F+(1-t) F^{\prime}$. We shall bound $\left|\frac{d}{d t} \log Z\left(\Lambda \mid F_{t}, b, \mu\right)\right|$ for $t=0$ or 1 .

Proceeding as above, and using the norm (3.18), this is bounded by

$$
\left\|F-F^{\prime}\right\| \cdot\left[Z^{-1} \sum_{\partial \subset \Lambda}^{0} e^{b V(\partial)} P(\partial)\left(\sum_{\Gamma \in \partial} e^{\tau \delta(\Gamma) / 2}\left(\theta(\Gamma)+\left\langle n\left(\omega_{\Gamma}\right)\right\rangle(\partial)\right)+O\left(e^{-\tau}\right)|\operatorname{Int} \Gamma|\right)\right],
$$

where we have used (A.31) for the estimate in $\operatorname{Int} \Gamma$. We shall prove below that

$$
\left\langle n\left(\omega_{\Gamma}\right)\right\rangle(\partial) \leqq O\left(\tau^{3}\right)|\Gamma|
$$

which is similar, but more subtle, than (A.34). [We could have used (A.35) in place of (A.34).]

Then we have,

$$
\sum_{\Gamma \in \partial}\left(\theta(\Gamma)+O\left(\tau^{3}\right)|\Gamma|+O\left(e^{-\tau}\right)|\operatorname{Int} \Gamma|\right) \leqq O\left(\tau^{3}\right)|\Lambda|
$$

for any $\partial$ made of outer contours in $\Lambda$. Moreover, if $\Gamma \subset \Lambda, \delta(\Gamma) \leqq \delta(\Lambda)-2(2 R+1)$, because $d(\Gamma, \bar{\Lambda})>2 R+1$. So we gain a factor $\exp (-\tau 2(2 R+1))$, which can "absorb" the $O\left(\tau^{3}\right)$ in (A.36) and give (3.21). So we are left with the proof of (A.35). Let $I(\cdot)$ denote a characteristic function and write

$$
\langle n\rangle=\langle n I(n \leqq A)\rangle+\langle n I(n \geqq A)\rangle \leqq A+e^{-(A / 2 \tau)} 2 \tau\langle\exp (n / \tau)\rangle
$$

[using $n \leqq 2 \tau \exp (n / 2 \tau)]$. 
Now, in $\langle\exp (n / \tau)\rangle$ we use (A.16) to "decouple" $\Gamma$ from the other contours, and then (3.13) on $\langle\exp (n / \tau)\rangle(\Gamma, F)$ as in the proof of (A.34). Thus we have

$$
\left\langle n\left(\omega_{\Gamma}\right)\right\rangle(\partial) \leqq A+2 \tau e^{-(A / 2 \tau)} \exp \left(\tau^{2}|\Gamma|+O\left(\varepsilon_{0}\right)|\Gamma|\right) .
$$

Choosing $A=2(\tau+1)^{3}|\Gamma|$ gives (A.35).

Acknowledgements. We thank R. Dobrushin, J. Imbrie, R. Kotecky, D. Martirosian, Y. Sinai, M. Zahradnik, and especially J. Slawny for interesting and useful discussions.

\section{References}

1. Peierls, R.: On Ising's model of ferromagnetism. Proc. Camb. Philos. Soc. 32, 477 (1936)

2. Dobrushin, R.L.: Existence of a phase transition in two- and three-dimensional lattice models. Theory Probab. Appl. 10, 193 (1965)

3. Griffiths, R.B.: Peierls' proof of spontaneous magnetization of a two-dimensional Ising ferromagnet. Phys. Rev. A 136, 437 (1964)

4. Ruelle, D.: Existence of a phase transition in a continuous classical system. Phys. Rev. Lett. 27, 1040 (1971)

5. Sinai, Ya.G.: Theory of phase transitions: Rigorous results. New York: Pergamon Press, 1982. Original papers: Pirogov, S.A., Sinai, Ya.G.: Phase diagram of classical lattice systems I and II: Theor. Math. Phys. 25, 1185 (1976) and 26, 39 (1976)

6. Pirogov, S.A., Sinai, Ya.G.: Ground states in two-dimensional Boson quantum field theory. Ann. Phys. 109, 393 (1977)

7. Imbrie, J.Z.: Phase diagrams and cluster expansions for low temperature $P(\phi)_{2}$ models I and II. Commun. Math. Phys. 82, 261 (1981) and 82, 305 (1981). For earlier results on phase transitions in quantum field theories, see: Glimm, J., Jaffe, A.: Quantum Physics. A functional integral point of view. New York: Springer 1981 (and references therein)

8. Slawny, J.: Low temperature expansion for lattice systems with many ground states. J. Stat. Phys. 26, 711 (1973). Low temperature properties of classical lattice systems: Phase transitions and phase diagrams. To appear in: "Phase transitions and critical phenomena," Vol. 10. Domb, C., Lebowitz, J.L. (eds.), New York: Academic Press 1985

9. Zahradnik, M.: An alternate version of Pirogov-Sinai theory. Commun. Math. Phys. 93, 559 (1984)

10. Kotecky, R., Preiss, D.: An inductive approach to Pirogov-Sinai theory. Proc. Winter School on Abstract Analysis 1983. Suppl. Ai. Rend Circ. Mat. Palermo (1983)

11. Malyshev, V.A., Minlos, R.A., Petrova, E.N., Teletski, A.: Generalized contour models (in Russian). Surveys of Science and Technology, pp. 3-54. Theory of probability, mathematical statistics and theoretical cybernetics, Vol. 19. Viniti 1982

12. Bricmont, J., Kuroda, K., Lebowitz, J.L.: The structure of Gibbs states and phase coexistence for non-symmetric continuum Widom-Rowlinson models. Z. Wahrscheinlichkeitstheor. Verw. Geb. 67, 121-138 (1984)

13. Dobrushin, R.L., Zahradnik, M.: Phase diagrams for the continuous spin models. Extension of Pirogov-Sinai theory. Preprint

14. Dinaburg, E.I., Sinai, Ya.G.: Preprint (in Russian); see also: "An analysis of ANNNI model by Peierls' contour method". Commun. Math. Phys. 98, 119 (1985)

15. Kotecky, R., Preiss, D.: In preparation

16. Kotecky, R., Shlosman, S.B.: First-order transitions in large entropy lattice models. Commun. Math. Phys. 83, 493 (1982)

17. Iagolnitzer, D., Souillard, B.: On the analyticity in the potential in classical statistical mechanics. Commun. Math. Phys. 60, 131 (1978), and references therein

18. Bricmont, J., Kuroda, K., Lebowitz, J.L.: Surface tension and phase coexistence for general lattice systems. J. Stat. Phys. 33, 59 (1983) 
19. Laanait, L., Messager, A., Ruiz, J.: Phase coexistence and surface tensions for the Potts model. Preprint

20. Bricmont, J., Lebowitz, J.L., P fister, C.-E.: Low temperature expansion for continuous spin Ising models. Commun. Math. Phys. 78, 117 (1980)

21. Widom, B., Rowlinson, J.S.: New model for the study of liquid-vapour phase transitions. J. Chem. Phys. 52, 1270 (1970)

22. Lebowitz, J.L., Penrose, O.: Rigorous treatment of the van der Waals-Maxwell theory of the liquid-vapour transition. J. Math. Phys. 7, 98 (1966)

23. Seiler, E.: Gauge theories as a problem of constructive quantum field theory and statistical mechanics. Lecture Notes in Physics, Vol. 159. Berlin, Heidelberg, New York: Springer 1982

Communicated by A. Jaffe

Received December 10, 1984; in revised form May 2, 1985 